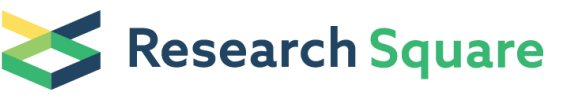

Preprints are preliminary reports that have not undergone peer review. They should not be considered conclusive, used to inform clinical practice, or referenced by the media as validated information.

\section{Elevated Exosome-derived Mirnas Predict Osimertinib Resistance in Non-small Cell Lung Cancer}

\section{Xinying Li}

Department of Respiratory and Critical Care Medicine, Jinling Hospital, Nanjing University School of Medicine, Nanjing, China. Nanjing University Institute of Respiratory Medicine, Nanjing, China

\section{Cen Chen}

Department of Respiratory Medicine, Jinling Hospital, the first School of Clinical Medicine, Southern Medical University (Guangzhou), Nanjing, China

\section{Zimu Wang}

Department of Respiratory and Critical Care Medicine, Jinling Hospital, Nanjing University School of Medicine, Nanjing, China. Nanjing University Institute of Respiratory Medicine, Nanjing, China

Jiaxin Liu

Department of Respiratory and Critical Care Medicine, Jinling Hospital, Nanjing University School of Medicine, Nanjing, China. Nanjing University Institute of Respiratory Medicine, Nanjing, China

\section{Wei Sun}

Department of Respiratory Medicine, Jinling Hospital, Medical School of Southeast University, Nanjing, China

\section{Kaikai Shen}

Department of Respiratory and Critical Care Medicine, Jinling Hospital, Nanjing University School of Medicine, Nanjing, China

\section{Yanling Lv}

Department of Respiratory and Critical Care Medicine, Jinling Hospital, Nanjing University School of Medicine, Nanjing, China

\section{Suhua Zhu}

Department of Respiratory and Critical Care Medicine, Jinling Hospital, Nanjing University School of Medicine, Nanjing, China

\section{Ping Zhan}

Department of Respiratory and Critical Care Medicine, Jinling Hospital, Nanjing University School of Medicine, Nanjing, China. Nanjing University Institute of Respiratory Medicine, Nanjing, China

\section{Tangfeng Lv}

Department of Respiratory and Critical Care Medicine, Jinling Hospital, Nanjing University School of Medicine, Nanjing, China. Nanjing University Institute of Respiratory Medicine, Nanjing, China

\section{Yong Song ( $\square$ yong.song@nju.edu.cn )}


Department of Respiratory Medicine, Jinling Hospital, Nanjing University School of Medicine https://orcid.org/0000-0003-4979-4131

\section{Research}

Keywords: Osimertinib (AZD9291), Exosome, miRNAs, Bypass pathway, NSCLC

Posted Date: December 8th, 2020

DOl: https://doi.org/10.21203/rs.3.rs-122187/v1

License: (c) (1) This work is licensed under a Creative Commons Attribution 4.0 International License. Read Full License 


\section{Abstract}

Background: Non-small cell lung cancer (NSCLC) patients with epidermal growth factor receptor (EGFR) mutations will inevitably develop drug resistance after being treated with the third-generation EGFRtyrosine kinase inhibitor (TKI), osimertinib. In recent years, many studies have been focusing on the ability of exosomal miRNAs secreted by tumor cells to transmit resistance information. However, the mechanism of exosome-derived miRNAs in osimertinib resistance remains unexplored.

Methods: We extracted and sequenced exosomes from the supernatant of the osimertinib-resistant cell line, $\mathrm{H} 1975-\mathrm{OR}$, and the sensitive cell line, $\mathrm{H} 1975$. The results were compared with plasma exosome sequencing before and after the appearance of drug resistance in three NSCLC clinical patients treated with oral osimertinib. Exosome-derived miRNAs that had significantly increased expression levels after osimertinib resistance were screened for expanded validation in other 64 NSCLC patients.

Results: Cluster analysis of the target genes revealed that exosomal miRNAs participate in osimertinib resistance mechanisms through the activation of bypass pathways (RAS-MAPK pathway abnormality and PI3K pathway activation). Exosome-derived miR-184 ( $p=0.0325)$ and miR-3913-5p $(p=0.0169)$ expression levels increased significantly after the onset of osimertinib resistance. Exosomal miR-184 was correlated with lactate dehydrogenase levels $(p=0.018)$. Exosomal miR-3913-5p was associated with TNM stage $(p=0.045)$, platelet count $(p=0.024)$, tumor marker carcinoembryonic antigen $(p=0.045)$, and distant metastases $(p=0.049)$, especially bone metastasis $(p=0.03)$. In the subgroup of patients with EGFR exon 21 L858R point mutation, miR-184 ( $p=0.0104)$ and miR-3913-5p ( $p=0.0085)$ derived from serum exosomes had both significantly increased expression levels. In the subgroup of T790Mpositive patients, miR-3913-5p derived from serum exosomes may also be a good indicator of osimertinib resistance $(p=0.013)$.

Conclusions: The expression levels of miR-184 and miR-3913-5p derived from exosomes in the peripheral blood of NSCLC patients could be used as biomarkers to indicate osimertinib resistance.

\section{Background}

Epidermal growth factor receptor (EGFR) mutations are the most common genetic mutations in non-small cell lung cancer (NSCLC)[1]. The PIONEER study (NCT01185314)[2] showed that the EGFR mutation rate in the Chinese population reached 50.2\%. Among them, exon 19 deletion (48.9\%) and exon 21 L858R point mutation (45.4\%) were the most common[2, 3]. Usually, $50-60 \%$ of the NSCLC patients with EGFR mutations develop acquired resistance to tyrosine kinase inhibitors (TKI) after first- and secondgeneration EGFR-TKI therapy[4], especially those with p.Thr790Met (T790M) point mutation in exon 20. The FLAURA study, which received wide attention at the 2019 European Society for Medical Oncology (ESMO) congress, confirmed that EGFR-mutant NSCLC patients treated with the third-generation EGFRTKI osimertinib[5] had a 6.8 months prolongation of overall survival (OS) compared to patients receiving gefitinib or erlotinib therapy[6]. However, patients treated with osimertinib inevitably develop resistance. 
Exosomes[7] are extracellular vesicles measuring 30-150 nm that transmit biological information and participate in cell-mediated biological activities by releasing large amounts of proteins, RNA, lipids, and other biomolecules to the extracellular environment[8]. MicroRNAs (miRNAs) [9] in exosomes are not digested by RNases, allowing them to be transported between cells and participate in epigenetics[10]. Existing literatures have shown that exosomal miRNAs (exo-miRNAs) secreted by tumor cells play an important role in drug resistance[11, 12]. Whether exosomes are involved in resistance to the thirdgeneration EGFR-TKI osimertinib remains unclear.

In this study, we investigated whether miRNAs in exosomes, especially before and after resistance to osimertinib, transmit relevant information between tumor cells, leading to changes in the sensitivity of patients. After collecting exosomes from the supernatant of osimertinib drug-resistant and drug-sensitive cells and comparing plasma exosomes of matched patients, a next-generation RNA-seq test was performed to identify key miRNAs. Later, we verified the role of key miRNAs in osimertinib resistance in larger population samples in an attempt to elucidate the mechanism of exo-miRNAs involved in osimertinib resistance. We hope to provide directions for the treatment of NSCLC patients with osimertinib resistance.

\section{Materials And Methods}

\section{Cell culture and establishment of resistant strains}

Lung adenocarcinoma cell line $\mathrm{H} 1975$ cells were purchased from the Institute of Biochemistry and Cell Biology, Chinese Academy of Sciences (Shanghai, China). Osimertinib was obtained from Selleck Chemicals (Houston, TX, USA). The osimertinib-resistant cell line, H1975-OR, was induced and cultivated as previously reported[13]. All cells were cultured in RPMI 1640 medium containing 10\% fetal bovine serum (FBS) and antibiotics (100 units/mL penicillin and $100 \mu \mathrm{g} / \mathrm{mL}$ streptomycin). All cells were incubated at $37^{\circ} \mathrm{C}$ in $5 \% \mathrm{CO}_{2}$ humidified air.

Cell proliferation was measured in three replicates using the MTT method. A non-linear regression function was used to fit the dose-response curve in GraphPad Prism 5.0. After the $I_{50}$ values of the semiinhibition rates of the two cells were obtained, the drug resistance index was calculated. Both MTT and dimethyl sulfoxide (DMSO) were purchased from Sigma-Aldrich (St. Louis, MO, USA). RPMI 1640 medium, fetal bovine serum (FBS), penicillin, and streptomycin were purchased from Gibco Life Technologies (Grand Island, NY, USA).

\section{Patient selection and sample collection}

A total of 67 NSCLC patients were included in this study and were observed in the Department of Respiratory and Critical Care Medicine at Jinling Hospital from December 2018 to October 2019. Three patients had a pair of blood samples collected each, before and after the appearance of resistance to osimertinib, to be compared with the $\mathrm{H} 1975$ cells samples. The samples of the remaining 64 patients were used for extended screening after identifying target miRNAs. Among these patients, 37 were 
resistant to gefitinib but had not yet developed osimertinib resistance, and 27 had Osimertinib resistance. In addition, 10 healthy people were recruited from the physical examination center of Jinling Hospital. All participants provided written informed consent. This study was approved by the ethics committee of Jinling Hospital.

\section{Isolation of exosomes}

After reaching $80 \%$ confluency in a Petri dish, $\mathrm{H} 1975$ and H1975-OR cells were cultured in complete RPMI1640 medium without added serum. The cell culture supernatant was collected $24 \mathrm{~h}$ later, and the exosomes were separated using differential ultracentrifugation as described previously[14]. To remove cell debris, the supernatant was centrifuged at $300 \times g$ for $5 \mathrm{~min}$, at $3000 \times g$ for $10 \mathrm{~min}$, and, lastly, at $10,000 \times g$ for $30 \mathrm{~min}$. The supernatant was then passed through a $0.22-\mu \mathrm{m}$ filter (Millipore, Burlington, MA, USA). The filtered supernatant was transferred to a clean ultracentrifuge tube and ultracentrifuged at $4{ }^{\circ} \mathrm{C}, 120000 \times \mathrm{g}$ for $70 \mathrm{~min}$. After discarding the supernatant, the pellet was resuspended in an appropriate amount of sterile phosphate-buffered saline $(1 \times \mathrm{PBS})$. The extracted exosomes were used for immediate downstream experiments or stored in a $-80^{\circ} \mathrm{C}$ refrigerator.

Serum samples were extracted using a Total Exosome Isolation Kit (from serum) (4478360, Invitrogen, Carlsbad, CA, USA,). The required volume of clear serum was transferred to a new test tube and 0.2 volume of total exosome isolation (from serum) reagent was added. The serum and reagent were mixed, through eddy current or an up-and-down pipe motion, to make the solution turbid. After incubation at $4{ }^{\circ} \mathrm{C}$ for $30 \mathrm{~min}$, the samples were centrifuged at $10,000 \times \mathrm{g}$ for $10 \mathrm{~min}$. The supernatant was aspirated and discarded. The exosomes, which were contained in the granules at the bottom of the test tube, were resuspended in half the volume of the initial serum volume of sterile PBS $(1 \times)$.

\section{Transmission electron microscopy (TEM), size distribution analysis, and western blot}

Exosome morphology was observed using TEM. The exosome suspension was mixed with an equal volume of $4 \%$ paraformaldehyde, and $10 \mu \mathrm{L}$ of the mixture was placed on a clean copper grid (RT) at room temperature. Uranyl acetate staining was negative. The images were acquired by observation with a JEOL 1200EX TEMSCAN microscope. The exosomal suspensions were analyzed for particle size using dynamic light scattering (DLS) (Nanosizer ${ }^{\mathrm{TM}}$ instrument, Malvern Instruments, Malvern, UK).

The extracted exosomes were resuspended in cell lysate (Beyotime, Nantong, China) supplemented with $1 \%$ PMSF. The protein concentration of exosomes was determined using a Pierce BCA protein detection kit (Thermo Fisher Scientific, Rockford, IL, USA). Twelve alkyl sulfate polyacrylamide gel electrophoresis were prepared with $20 \mu \mathrm{g}$ of protein on each sample. Anti-CD63, anti-TSG101, anti- $\beta$-actin, and antiGAPDH were purchased from Abcam (Cambridge, UK).

\section{RNA-sequencing and raw data}


The total RNA in the exosomes was extracted using TRIzol reagent (Invitrogen, Carlsbad, CA, USA) according to the manufacturer's instructions. After performing the RNA extraction and quality control, the library was constructed and sequenced according to the literature. The raw data from small RNA-seq include linker sequences and sequencing low-quality sequences. In order to ensure the accuracy of the information analysis, the raw sequencing data were filtered to obtain clean data, and subsequent bioinformatics analysis was performed based on the clean data. The Qphred score (Qphred $=10 \log 10$ (e)) was used to represent the base quality value (Quality Score) to measure the quality of each base in the sequencing reads. The miRDeep2 software was used to analyze miRNA expression abundance. Cluster analysis and correlation analysis between samples (Pearson's correlation coefficient) were performed on the miRNA family in each sample. All raw sequencing data were uploaded to the SRI database. [Submission ID: SUB7187450; BioProject ID: PRJNA615931] (http://www.ncbi.nlm.nih.gov/bioproject/615931)

\section{Target gene analysis}

The analysis was performed using DESeq2 (no biological duplicate samples use DESeq or edgeR); miRNAs with [logFoldChage] $>1$ and $p$ value $<0.05$ were selected as miRNAs with significant differences. A volcano map of the results of miRNA differential analysis and the clustered heat map of miRNA expression in the samples were drawn. TargetScan was used to calculate a weighted context ++ score to predict the target genes of different known miRNAs. These target gene functions were classified through a database established using the Gene Ontology (GO) Consortium, and a GO enrichment analysis was performed. At the same time, the first 10 target genes of each sample were selected for Kyoto Encyclopedia of Genes and Genomes (KEGG) pathway enrichment analysis according to the parameters described in the literature to identify the downstream molecular metabolic pathways of these target genes.

\section{Quantitative reverse transcription PCR}

The RNA extracted from the sera of patients was quantified and evaluated using NanoDrop ${ }^{\circledR}$ ND-2000 (Thermo Fisher Scientific, USA). We used the miRNA first-strand cDNA synthesis (tailing method) kit from Shanghai Sangon Biotech, using the method of Poly $(A)$ tailing reaction and cDNA synthesis reaction simultaneously. After following the manufacturer's instructions, all cDNA products of the miRNAs were obtained and real-time PCR experiments were performed. In the RT-PCR, the universal downstream primer Universal PCR Primer R and the endogenous reference U6 (Universal U6 Primer F) in the above kit were used uniformly. The upstream primers of several miRNAs are as follows: miR-184: 5'catGGACGGAGAACtGAtAAGGGt-3 '; miR-3913-5p: 5'-acggTTTGGGACTGATCTTGATGTCT-3'; miR-4746-5p: 5'-CCGGTCCCAGGAGAACC-3'; miR-3614-5p: 5'-CCACTTGGATCTGAAGGCTGC-3'. RT-PCR was performed using an $A B I 7500$ real-time PCR system (Applied Biosystems, Foster City, CA, USA). The expression levels of all miRNAs were determined using the $2-\triangle \Delta C T$ method: $\triangle C T$ (target) $=C T$ (target) $-C T$ (U6). All the above experiments were performed twice.

\section{Statistical analysis}


The above data were mostly analyzed and mapped using the SPSS 22.0 system (SPSS, Inc. Chicago, IL, USA) and GraphPad Prism 5. The differences in the miRNA expression levels between the two groups were mainly determined using the Mann-Whitney rank-sum test for non-parametric data. The correlation between Exo-miR-184 and Exo-miR-3913-5p with clinicopathological features was determined using the $\chi 2$ test. ROC curves were also used to determine the diagnostic value. $P>0.05$ values indicate a statistical difference.

\section{Results}

\section{Isolation of exosomes from cell supernatant and serum}

In the commonly used lung cancer cell lines, H1975 carries an EGFR-L858R drug-sensitive mutation or EGFR-T790M drug-resistant mutation. The drug-resistant strain H1975-OR was established by increasing the concentration of osimertinib. Six months later, the MTT method was used to determine the cell viability of the sensitive strain $\mathrm{H} 1975$ and the resistant strain H1975-OR (Fig. 1A). The average $\mathrm{IC}_{50}$ values of these two cells were $4636 \mathrm{nM}$ and $12101 \mathrm{nM}$, respectively ( $p=0.0215)$ (Fig. 1B); RI was 2.61. Circular vesicle-like exosomes were obtained from different cell supernatants after ultracentrifugation. Blood samples were collected from patients with EGFR mutations before and after osimertinib resistance. The exosomes were extracted using the kit. Exosomes were also identified using TEM (Fig. 1C), western blotting (Fig. 1D), and DLS analysis (Fig. 1E, F).

\section{Functional enrichment analysis of differentially expressed miRNA target genes in exosomes}

The volcano map visually revealed the difference in miRNA expression of exosomes in the supernatant of H1975 and H1975-OR cells (Fig. 2A). Among them, the expression levels of exosome miR-6087, miR-99b$5 p$, and miR-7641 in the H1975-OR supernatant were significantly higher than those in the H1975 supernatant. Other exo-miRNAs, such as miR-378a-3p, miR-25-5p, and miR-1293, were significantly reduced (Fig. 2B). KEGG pathway enrichment was carried out for the top ten target genes of exosome miRNAs with differences between the two groups. The "metabolic pathway" involved the largest number of target genes, followed by the "PI3K-Akt signaling pathway," "Ras signaling pathway," "cytokine-cytokine receptor interaction," "non-small cell lung cancer," and other pathways (Fig. $2 \mathrm{C}$ ). The pathway analysis of novel differentially expressed miRNA target genes focused on the MAPK signaling pathway (Supplementary Fig. 1). These pathways are also members of the bypass pathways involved in drug resistance in EGFR-TKI therapy for NSCLC.

A 62-year-old male with NSCLC (Patient 2, Treat3, \& Contro/3) was treated with gefitinib after molecular testing, which revealed an EGFR exon 19 deletion mutation. One year later, he started oral AZD9291 due to new brain and bone metastasis. The cancer progressed after 19 months. Blood samples were collected from the patient at the start of osimertinib treatment and after presenting resistance. Similarly, blood samples were collected from a 53-year-old female patient (left lung adenocarcinoma stage IVB, EGFR 21 
L858R mutation) (Patient 1, Treat2, \& Contro/2) and a 58-year-old female patient (left lung adenocarcinoma stage IVB, EGFR 19 deletion) (Patient 3, Treat4, \& Contro/4) before and after the appearance of resistance to osimertinib. Pearson's correlation coefficient (R2) was used to assess the correlation between exo-miRNAs from the cell supernatant and serum of patients, and the correlation heat map matrix was drawn (Fig. 2D).

\section{Elevated miR-184 and miR-3913-5p in exosomes after osimertinib resistance}

We performed an overlapping analysis of the differential miRNAs obtained by sequencing exosomes in the cultured cells supernatant (treat1) and the blood samples of three patients (treat2-4) using a Wayne map (Fig. 3A, B). Among the exo-miRNAs upregulated in the osimertinib-resistant group compared to those in the sensitive group, no common differential miRNAs were found to overlap in the four groups (Fig. 3A). However, in a pairwise comparison, it was found that miR-184 overlapped in treat1 and treat4, miR-3913-5p appeared both in treat3 and treat4, and miR-3656 co-occurred in treat1 and treat2 (Fig. $3 \mathrm{~A}$ ). These three miRNAs were further studied. Similarly, miR-3614-5p, miR-4746-5p, and miR-378i were chosen as the target miRNAs in the downregulation group (Fig. 3B).

In order to verify several significantly up-regulated exo-miRNAs in the RNA-seq results, we decided to expand the sample size for experiments. Serum samples from 37 NSCLC patients with gefitinib resistance and 27 patients with osimertinib resistance were collected as controls (GR) and experimental groups (OR) before and after osimertinib resistance. Using QPCR, we verified that the exosome miR-184 in the serum of drug-resistant patients was significantly high ( $p=0.0325)$ (Fig. $3 \mathrm{C}$ ), and exosome miR-3913$5 p$ was significantly increased in the osimertinib-resistant group $(p=0.0169)$ (Fig. 3D), which was consistent with the previous sequencing results.

\section{Increased miRNAs suggested that NSCLC patients were resistant to osimertinib}

Combining these two exo-miRNAs, it was found that these two miRNAs in the serum exosomes of NSCLC patients were significantly upregulated after osimertinib resistance $(p=0.0092)(F i g .3 E, F)$. The relationship between exo-miRNA expression levels and the clinicopathological characteristics of the patients (e.g., age, sex, smoking history, TNM stage, primary tumor size, platelet count (PLT), lactate dehydrogenase (LDH), carcinoembryonic antigen (CEA), and distant metastasis) is summarized in Tables 1 and 2. Exosome miR-184 was correlated with LDH levels $(p=0.018)($ Table 1$)$; exosome miR3913-5p was associated with TNM stage $(p=0.045)$, PLT $(p=0.024)$, tumor marker CEA $(p=0.045)$, distant metastases $(p=0.049)$, and, especially, bone metastasis $(p=0.03)$ (Table 2$)$. However, there was no significant correlation between miRNA expression levels of the two exosomes and age, sex, smoking status, ECOG score, and primary tumor size. 
Table 1

Correlation between serum exosomal miR-184 expression levels in patients with NSCLC and their clinicopathological characteristics. $\left({ }^{*} p<0.05\right)$

\begin{tabular}{|c|c|c|c|c|}
\hline & & Exosc & nal mi & -184 \\
\hline Clinicopathological parameters & Number of patients(\%) & Low & High & $P$ \\
\hline Age(years), Median & & & & 0.777 \\
\hline$₫ 60$ & 33 & 15 & 18 & \\
\hline$\geq 60$ & 31 & 13 & 18 & \\
\hline Gender & & & & 0.899 \\
\hline Male & 28 & 12 & 16 & \\
\hline Female & 36 & 16 & 20 & \\
\hline Smoking history & & & & 0.353 \\
\hline Never smoking & 49 & 23 & 26 & \\
\hline Now/once smoking & 15 & 5 & 10 & \\
\hline ECOG Score & & & & 0.226 \\
\hline $0-1$ & 53 & 25 & 28 & \\
\hline$\geq 2$ & 11 & 3 & 8 & \\
\hline Primary tumor & & & & 0.705 \\
\hline$\leq 4 \mathrm{~cm}$ & 19 & 9 & 10 & \\
\hline$\nabla 4 \mathrm{~cm}$ & 45 & 19 & 26 & \\
\hline TNM & & & & 0.317 \\
\hline I-IVA & 25 & 9 & 16 & \\
\hline IVB & 39 & 19 & 20 & \\
\hline PLT & & & & 0.411 \\
\hline $\operatorname{Low}(\leq 146)$ & 13 & 7 & 6 & \\
\hline 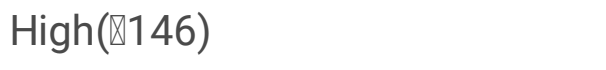 & 51 & 21 & 30 & \\
\hline LDH & & & & 0.018 * \\
\hline $\operatorname{Low}(\leq 199)$ & 29 & 8 & 21 & \\
\hline 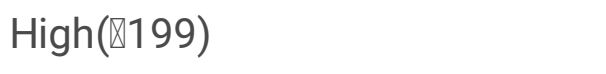 & 35 & 20 & 15 & \\
\hline CEA & & & & 0.129 \\
\hline
\end{tabular}




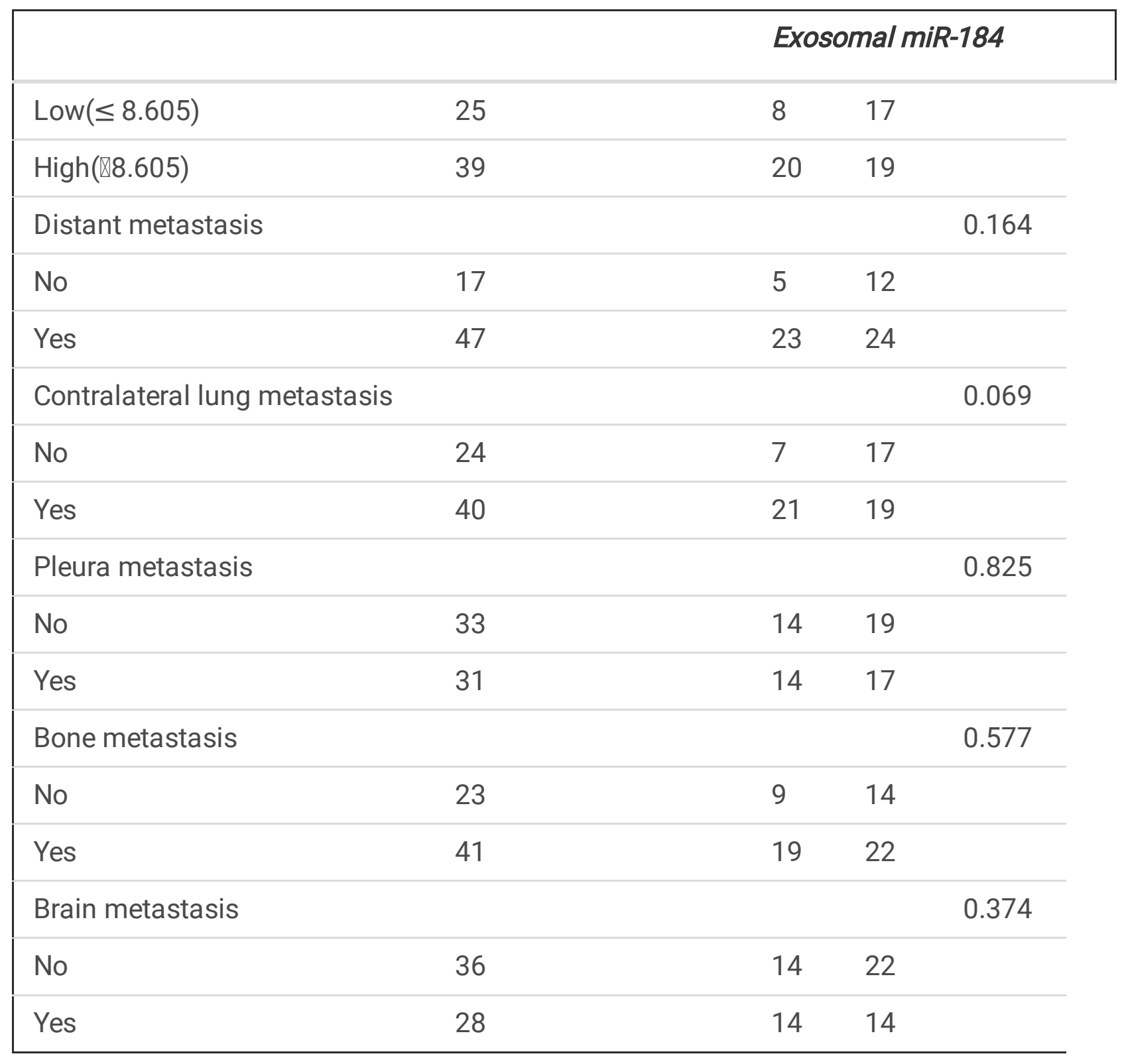


Table 2

Correlation between serum exosomal miR-3913-5p expression levels in patients with NSCLC and their clinicopathological characteristics.

\begin{tabular}{|c|c|c|c|c|}
\hline \multirow[b]{2}{*}{ Clinicopathological parameters } & \multirow[b]{2}{*}{ Number of patients(\%) } & \multicolumn{3}{|c|}{ Exosomal miR-3913-5p } \\
\hline & & Low & High & $P$ \\
\hline Age(years), Median & & & & 0.714 \\
\hline$\llbracket 60$ & 32 & 25 & 7 & \\
\hline$\geq 60$ & 31 & 23 & 8 & \\
\hline Gender & & & & 0.427 \\
\hline Male & 28 & 20 & 8 & \\
\hline Female & 35 & 28 & 7 & \\
\hline Smoking history & & & & 0.321 \\
\hline Never smoking & 48 & 38 & 10 & \\
\hline Now/once smoking & 15 & 10 & 5 & \\
\hline ECOG Score & & & & 0.064 \\
\hline $0-1$ & 52 & 42 & 10 & \\
\hline$\geq 2$ & 11 & 6 & 5 & \\
\hline Primary tumor & & & & 0.64 \\
\hline$\leq 4 \mathrm{~cm}$ & 18 & 13 & 5 & \\
\hline$\nabla 4 \mathrm{~cm}$ & 45 & 35 & 10 & \\
\hline TNM & & & & $0.045^{*}$ \\
\hline I-IVA & 24 & 15 & 9 & \\
\hline IVB & 39 & 33 & 6 & \\
\hline PLT & & & & $0.024 *$ \\
\hline $\operatorname{Low}(\leq 146)$ & 13 & 13 & 0 & \\
\hline 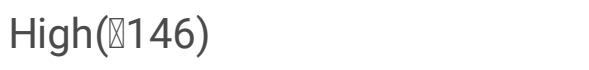 & 50 & 35 & 15 & \\
\hline LDH & & & & 0.427 \\
\hline $\operatorname{Low}(\leq 199)$ & 28 & 20 & 8 & \\
\hline 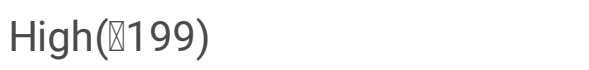 & 35 & 28 & 7 & \\
\hline$\left({ }^{*} p<0.05\right)$ & & & & \\
\hline
\end{tabular}




\begin{tabular}{|c|c|c|c|c|c|}
\hline & & & \multicolumn{3}{|c|}{ Exosomal miR-3913-5p } \\
\hline CEA & & & & & $0.045^{*}$ \\
\hline $\operatorname{Low}(\leq 8.605)$ & 24 & & 15 & 9 & \\
\hline $\operatorname{High}(\llbracket 8.605)$ & 39 & & 33 & 6 & \\
\hline Distant metastasis & & & & & $0.049 *$ \\
\hline No & 17 & & 10 & 7 & \\
\hline Yes & 46 & & 38 & 8 & \\
\hline Contralateral lung metastasis & & & & & 0.434 \\
\hline No & 24 & & 17 & 7 & \\
\hline Yes & 39 & & 31 & 8 & \\
\hline Pleura metastasis & & & & & 0.414 \\
\hline No & 32 & & 23 & 9 & \\
\hline Yes & 31 & & 25 & 6 & \\
\hline Bone metastasis & & & & & 3* \\
\hline No & 23 & 14 & & & \\
\hline Yes & 40 & 34 & & & \\
\hline Brain metastasis & & & & & 112 \\
\hline No & 35 & 24 & & & \\
\hline Yes & 28 & 24 & & & \\
\hline$\left({ }^{*} p<0.05\right)$ & & & & & \\
\hline
\end{tabular}

Exosomal-derived miRNAs predict osimertinib resistance in patients with EGFR exon21 L858R mutation.

Of the 64 NSCLC patients, 28 possessed EGFR exon 19 deletions and 36 had mutations in the EGFR exon 21. In all patients with exon 19 deletion, there was no significant difference in the expression levels of serum exosomal miR-184 $(p=0.776)$ and miR-3913-5p $(p=0.631)$ between the groups before and after drug resistance (Supplementary Fig. 3A, B). However, in all patients with mutations in L858R in the EGFR exon 21, the serum expression level of exo-miR-184 was significantly elevated in osimertinib-resistant patients $(p=0.0104)$ (Fig. 4A). Exo-miR-3913-5p ( $p=0.0085)$ was also significantly altered in patients with osimertinib resistance (Fig. 4B). ROC curve analysis showed that the AUC of exo-miR-184 was 0.736, while the AUC of exo-miR-3913-5p was 0.759 (Supplementary Fig. 3C, D), indicating that these two miRNAs play important roles in osimertinib resistance and were mainly involved in drug resistance in patients with EGFR exon 21 L858R. Because the sensitive group (GR) was selected for gefitinib-resistant 
patients, the criteria for clinical resistance in these patients were enlarged lesions or new organ metastases. After re-sequencing, most patients $(n=44)$ showed T790M-positive mutations in EGFR-TKI resistance, but a few patients $(n=20)$ were not T790M-positive (Supplementary Fig. 3). In T790M + osimertinib-resistant patients, the expression level of exo-miR-3913-5p was significantly increased $(p=$ 0.013) (Fig. 4D), and the exo-miR-184 expression level in the resistant group was also higher than that in the sensitive group, but the difference was not statistically significant $(p=0.065)$ (Fig. 4C).

\section{Discussion}

Osimertinib (AZD9291) is the first third-generation EGFR-TKI to be approved by the FDA and EMA for the treatment of NSCLC[5]. It is selective for EGFR-TKI sensitization and T790M resistance mutations and has less effect on WT-EGFR[15]. However, similar to the findings observed after the use of other EGFRTKIs, patients still inevitably develop resistance after receiving osimertinib, regardless of its first- or second-line use, which greatly limits the long-term clinical benefits of this targeted drug[16]. The mechanism of osimertinib resistance depends on the high tumor heterogeneity of NSCLC, which is divided into EGFR-dependent and EGFR-independent aspects[17]. Moreover, previous studies have shown that the resistance mechanism after first- or second-line use of osimertinib varies with clonal evolution[18]. Our study focused on patients with advanced NSCLC, who were treated with non-first-line osimertinib. Patients enrolled in the sensitive group (GR) were those who were sensitive to osimertinib after gefitinib resistance, while patients in the resistant group (OR) were those who had developed clinical resistance to osimertinib. This ensured the homogeneity of these two sample groups.

The most common EGFR-dependent drug-resistance mechanism is C797S mutation occurring on exon 20 , which has previously been reported to account for $10-26 \%$ of all cases using osimertinib as secondline therapy[19]. In addition to C797S-adjacent G796 mutation, there are multiple mutation sites possibly involved, which include L792, L718, G719, G724, and EGF overexpression[20]. However, in our study, of the 27 patients with osimertinib resistance, only $3(11.11 \%)$ had a C797S mutation that was confirmed based on molecular testing. Of course, there were also several patients who did not undergo genetic testing again for financial reasons.

The most important EGFR-independent mechanisms of drug resistance are activation of bypass signals, abnormalities in downstream pathways, and histological transformation. The most common activated bypass pathways are MET amplification[21] and HER2 amplification[22]. The abnormality of the RASMAPK pathway is also an important mechanism of osimertinib resistance. Ortiz-Cuaran et al.[23] confirmed that when acquired resistance to second-line osimertinib was developed, re-biopsy of the tumor revealed a KRAS G12S mutation. Kim et al.[24] reported a case of MAPK1 mRNA overexpression in a patient who received second-line treatment with osimertinib in advanced stages. This is consistent with our exosome sequencing results. As shown in Fig. 2C, the most obvious pathway enriched by the known differential exo-miRNA target gene in the supernatants of drug-resistant (H1975-OR) and sensitive (H1975) strains was the Ras signaling pathway. As shown in Supplementary Fig. 1, the MAPK signaling pathway is the most abundant among the novel differential miRNA target genes with the largest number 
of genes involved. The above results indicated that the participation of exo-miRNAs in osimertinib resistance was also related to abnormalities in the RAS-MAPK pathway. In addition, the PI3K pathway is involved in bypass activation. It is currently believed that PIK3CA mutation or amplification and PTEN deletion can lead to activation of the PI3K pathway[24]. In our study, the PI3K-Akt signaling pathway was observed in KEGG enrichment analysis. Exo-miRNAs may convey osimertinib resistance information to affect the activation of the PI3K pathway. Osimertinib resistance is also related to changes in cell cycle genes, including cyclin D1, cyclin D2, cyclin E1, cyclin-dependent kinase (CDK) 4, and CDK6[25]. In the sequencing results, exosome miR-6087 was significantly increased in the drug-resistant group. Its target gene was CCND1 (Supplementary Table 1), which encodes the cyclin D1 protein. This again demonstrated that exosomes participated in osimertinib resistance by having an impact on bypass activation.

As mentioned previously, exosomes can contain a large number of proteins, nucleic acids, and lipids, which transmit information between cells[26]. Tumor-derived exosomes can be detected in the blood and body fluids of patients. It has been demonstrated that exosomes can affect the therapeutic response and induce drug resistance in tumor cells[27]. Recent research has suggested that drug-resistant cells transmit drug resistance information to drug-sensitive cells through extracellular vesicles[28]. The miRNAs, which are short non-coding RNA, have been thoroughly studied in oncology[29]. When miRNAs are loaded into exosomes, they can be protected from degradation by RNases[30]. An interesting finding was the discovery that exo-miRNAs could assist in the diagnosis of NSCLC. We compared the levels of exosome miR-184, miR-3913-5p, miR-3614-5p, and miR-4746-5p in the serum of NSCLC patients and healthy individuals, and found significant differences between the two. The area under the curve (AUC) of miR-184 was 0.803 (95\% confidence interval: $0.701-0.905)$, greater than 0.75 , indicating that miR-184 of serum exosomes of lung cancer patients could be used as a biomarker for the diagnosis of NSCLC (Supplementary Fig. 4).

In recent years, many papers have been published on the relationship between exosome derived miRNAs and drug resistance in the treatment of NSCLC[31, 32]. However, few studies have discussed the relationship between the resistance to the third-generation EGFR-TKI osimertinib and exosomes. In our study, we observed exosomes extracted from the cultured H1975 and H1975-OR cell supernatant and the serum of three patients. Changes in the expression levels of exo-miRNAs after osimertinib resistance were captured. We identified, for the first time, that the increased levels of miR-184 and miR-3913-5p can predict resistance to osimertinib in NSCLC patients treated with this drug. In the clinicopathological features, LDH has been proven to be closely related to clinical prognosis in a variety of malignant tumors[33]. NSCLC patients with higher LDH levels have a worse prognosis and shorter survival than those with lower levels[34]. Although the exosome miR-184 found in our study was related to LDH level ( $p$ $=0.018$ ) because of the time limit of this experiment, we could not continue to follow up to obtain survival data; therefore, whether exosome miR-184 could be a prognostic indicator remains unclear. CEA is the most common biomarker of lung adenocarcinoma[35]. It has been confirmed that an increase in CEA levels during TKI treatment for EGFR mutation patients may be a more sensitive predictor of an explosive progression in lung adenocarcinoma[36]. PLT is often associated with the platelet-to- 
lymphocyte ratio (PLR)[37]. Studies have suggested that the preoperative PLT-PLR score could be of significance in predicting the prognosis of patients with surgically resected NSCLC[38]. In this study, we found that exo-miR-3913-5p was related to TNM stage $(p=0.045), \operatorname{PLT}(p=0.024)$, CEA $(p=0.045)$, distant metastasis $(p=0.049)$, and bone metastasis $(p=0.03)$. Moreover, the AUC in the receiver operating characteristic curve was greater than 0.75 , which further suggested that the exosome miR3913-5p expression level was associated with advanced progression of lung adenocarcinoma in patients with EGFR mutations during TKI use.

Venn diagram analysis demonstrated that miR-184 expression level alteration was overlapped in Treat1 (H1975 lines cells) and Treat4 (A patient with EGFR exon 19 del). MiR-3913-5p expression level alteration was found to be overlapped in Treat3 and Treat4, and both patients had EGFR exon 19 deletions. However, we found in these subgroup analyses that the expression levels of both miRNAs were significantly altered in patients with EGFR exon 21 L858R point mutations. Previous studies have found that exon 19 deletion mutations (55.0\%) have a higher rate of T790M resistance mutations than exon 21 L858R point mutations (37.3\%)[39]. Recent studies have shown that the hazard ratio of survival benefit for Asian and L858R mutant populations is close to 1.00 in all people receiving osimertinib treatment[6]. Our study found that the expression levels of these two exo-miRNAs changed significantly in the L858R mutant population; however, this phenomenon was not observed in the population with exon 19 deletion. Thus, these two exosome-derived miRNAs might be more useful to predict resistance to osimertinib in patients with EGFR exon 21 L858R point mutations than in other populations. Previous studies have reported that nearly half of the patients lost the T790M mutation at the time of progression to osimertinib[40], and this loss may be related to the early resistance to osimertinib. The loss of T790M mutation is not conducive to prognosis[41]. Plasma T790M levels may predict acquired resistance[42]. However, considering that osimertinib is selective for EGFR sensitivity and T790M mutation, scholars believe that the emergence of a T790M mutation under osimertinib treatment is not a drug-resistance mechanism[17]. However, in this study, we found that exosomal miR-3913-5p expression levels were significantly altered in T790M-positive patients, indicating that this exo-miRNA may be involved in the drug-resistance mechanism in such patients. Exosomal miR-184 and miR-3913-5p are likely to be important molecules for the transmission of osimertinib resistance.

Our study had some limitations. The number of patients validated in this study was 64 , and the number of collected specimens was not ideal. Only patient serum samples were used to extract exosomes, and no further humoral exosomes were used for verification. The clinical characteristics of the patients were collected, but no survival analysis was performed owing to the limitation of follow-up time. The predicted target genes and pathways will be verified in our subsequent experiments.

Our experiment demonstrated that exosome-derived miRNAs may be involved in the mechanisms of resistance to the third-generation EGFR-TKI osimertinib, especially affecting bypass pathway activation. Therefore, an increase in the expression levels of exosome-derived miR-184 and miR-3913-5p in the peripheral blood of NSCLC patients receiving osimertinib treatment may indicate the development of drug resistance. We hope that this study helps to expand the application of liquid biopsy technology in the 
field of clinical drug resistance in lung cancer. This may not only favorably guide clinical treatment but also provide direction for the development of a new generation of targeted drugs.

\section{List Of Abbreviations}

NSCLC, non-small cell lung cancer; TKI, tyrosine kinase inhibitor; EGFR, epidermal growth factor receptor; BRAF, serine/threonine protein kinase b-Raf; T790M, Thr790Met; DMSO, dimethyl sulfoxide; FBS, fetal bovine serum; PLT, platelet count ; LDH, lactate dehydrogenase; CEA, carcinoembryonic antigen.

\section{Declarations}

\section{Ethics approval and consent to participate}

The research protocol was reviewed and approved by the Ethical Committee and Institutional Review Board of the Jinling Hospital affiliated to Nanjing University School of Medicine, and written informed consent was obtained from each patient included in the study.

\section{Consent for publication}

Not applicable.

\section{Availability of data and materials}

The datasets used and/or analyzed during this study are available from the corresponding author upon reasonable request.

\section{Competing interests}

The authors declare no conflicts of interest.

\section{Funding}

This work was supported by grants from the National Natural Science Foundation of China (grant numbers 81772500,81770082 , and 81572273) and the Key Research and Development Program of Jiangsu Province (grant number BK2019719).

\section{Authors' contributions}

X. Li and T. Lv elaborated the initial experimental design. C. Chen completed the design of the overall experimental scheme. X. Li wrote the first draft of the article. Z. Wang, J. Liu, and W. Sun completed the drawing of pictures and tables. M. Chen participated in the extraction of exosomes. X. Li, C. Chen, and S. Zhu completed exosome identification and extended sample validation experiments. K. Shen and Y. LV conducted the data analysis. P. Zhan conceived the project. T. Lv and Y. Song supervised the research. All authors edited the final manuscript together. 


\section{Acknowledgments}

The authors would like to extend their sincere gratitude to the reviewers.

\section{References}

1. Takano, T. Epidermal Growth Factor Receptor Gene Mutations and Increased Copy Numbers Predict Gefitinib Sensitivity in Patients With Recurrent Non-Small-Cell Lung Cancer. Journal of Clinical Oncology.23(28):6829-37.

2. Shi Y, Li J, Zhang S, Wang M, Yang S, Ning L, et al. Molecular Epidemiology of EGFR Mutations in Asian Patients with Advanced Non-Small-Cell Lung Cancer of Adenocarcinoma Histology \&ndash; Mainland China Subset Analysis of the PIONEER study. Plos One. 2015;10(11):e0143515.

3. Kumarakulasinghe NB, van Zanwijk N, Soo RA. Molecular targeted therapy in the treatment of advanced stage non-small cell lung cancer (NSCLC). Respirology (Carlton, Vic). 2015;20(3):370-8.

4. Maemondo M, Inoue A, Kobayashi K, Sugawara S, Oizumi S, Isobe H, et al. Gefitinib or chemotherapy for non-small-cell lung cancer with mutated EGFR. The New England journal of medicine. 2010;362(25):2380-8.

5. Cross DA, Ashton SE, Ghiorghiu S, Eberlein C, Nebhan CA, Spitzler PJ, et al. AZD9291, an irreversible EGFR TKI, overcomes T790M-mediated resistance to EGFR inhibitors in lung cancer. Cancer discovery. 2014;4(9):1046-61.

6. Ramalingam SS, Vansteenkiste J, Planchard D, Cho BC, Gray JE, Ohe Y, et al. Overall Survival with Osimertinib in Untreated, EGFR-Mutated Advanced NSCLC. The New England journal of medicine. 2020;382(1):41-50.

7. Schorey JS, Bhatnagar S. Exosome function: from tumor immunology to pathogen biology. Traffic (Copenhagen, Denmark). 2008;9(6):871-81.

8. van der Pol E, Boing AN, Harrison P, Sturk A, Nieuwland R. Classification, functions, and clinical relevance of extracellular vesicles. Pharmacological reviews. 2012;64(3):676-705.

9. Valadi H, Ekstrom K, Bossios A, Sjostrand M, Lee JJ, Lotvall JO. Exosome-mediated transfer of mRNAs and microRNAs is a novel mechanism of genetic exchange between cells. Nature cell biology. 2007;9(6):654-9.

10. Falcone G, Felsani A, D'Agnano I. Signaling by exosomal microRNAs in cancer. Journal of experimental \& clinical cancer research : CR. 2015;34:32.

11. Yu DD, Wu Y, Zhang XH, Lv MM, Chen WX, Chen X, et al. Exosomes from adriamycin-resistant breast cancer cells transmit drug resistance partly by delivering miR-222. Tumour biology : the journal of the International Society for Oncodevelopmental Biology and Medicine. 2016;37(3):3227-35.

12. Alharbi M, Zuniga F, Elfeky O, Guanzon D, Lai A, Rice GE, et al. The potential role of miRNAs and exosomes in chemotherapy in ovarian cancer. Endocrine-related cancer. 2018;25(12):R663-r85. 
13. Li XY, Huang LT, Wu JQ, He MF, Zhu SH, Zhan P, et al. Zebrafish Xenograft Model of Human Lung Cancer for Evaluating Osimertinib Resistance. BioMed research international. 2019;2019:3129748.

14. Théry C, Amigorena S, Raposo G, Clayton A. Current Protocols in Cell Biology. Current protocols in cell biology / editorial board, Juan S Bonifacino [et al]. 2006;Chapter 3:Unit 3.22.

15. Minari R, Bordi P, Tiseo M. Third-generation epidermal growth factor receptor-tyrosine kinase inhibitors in T790M-positive non-small cell lung cancer: review on emerged mechanisms of resistance. Translational Lung Cancer Research. 2016;5(6):695-708.

16. Neel DS, Bivona TG. Resistance is futile: overcoming resistance to targeted therapies in lung adenocarcinoma. npj Precision Oncology. 2017;1(1):3.

17. Leonetti A, Sharma S, Minari R, Perego P, Giovannetti E, Tiseo M. Resistance mechanisms to osimertinib in EGFR -mutated non-small cell lung cancer. British Journal of Cancer. 2019;121:1-13.

18. Bollinger M, Agnew A, Mascara G. Osimertinib: A third-generation tyrosine kinase inhibitor for treatment of epidermal growth factor receptor-mutated non-small cell lung cancer with the acquired Thr790Met mutation. Journal of Oncology Pharmacy Practice. 2017;24:107815521771240.

19. Mok T, Wu Y-L, Ahn M-J, Garassino M, Kim HR, Ramalingam S, et al. Osimertinib or PlatinumPemetrexed in EGFR T790M-Positive Lung Cancer. New England Journal of Medicine. 2016;376.

20. Yang Z, Yang N, Ou Q, Xiang Y, Jiang T, Wu X, et al. Investigating Novel Resistance Mechanisms to Third-Generation EGFR Tyrosine Kinase Inhibitor Osimertinib in Non-Small Cell Lung Cancer Patients. Clinical Cancer Research. 2018;24:clincanres.2310.017.

21. Yu HA, Arcila ME, Rekhtman N, Sima CS, Zakowski MF, Pao W, et al. Analysis of Tumor Specimens at the Time of Acquired Resistance to EGFR-TKI Therapy in 155 Patients with <em>EGFR</em>-Mutant Lung Cancers. Clinical Cancer Research. 2013;19(8):2240-7.

22. Planchard D, Loriot Y, Andre F, Gobert A, Auger N, Lacroix L, et al. EGFR independent mechanisms of acquired resistance to AZD9291 in EGFR T790M-positive NSCLC patients. Annals of oncology : official journal of the European Society for Medical Oncology / ESMO. 2015;26.

23. Ortiz-Cuaran S, Scheffler M, Plenker D, Dahmen I, Scheel A, Fernández-Cuesta L, et al. Heterogeneous Mechanisms of Primary and Acquired Resistance to Third-Generation EGFR Inhibitors. Clinical Cancer Research. 2016;22.

24. Kim T, Song A, Kim D-W, Kim S, Ahn Y-O, Keam B, et al. Mechanisms of Acquired Resistance to AZD9291, a Mutation-Selective, Irreversible EGFR Inhibitor. Journal of thoracic oncology : official publication of the International Association for the Study of Lung Cancer. 2015;10.

25. Blakely CM, Watkins TBK, Wu W, Gini B, Chabon JJ, McCoach CE, et al. Evolution and clinical impact of co-occurring genetic alterations in advanced-stage EGFR-mutant lung cancers. Nature Genetics. 2017;49(12):1693-704.

26. Regev-Rudzki N, Wilson DW, Carvalho TG, Sisquella X, Coleman BM, Rug M, et al. Cell-cell communication between malaria-infected red blood cells via exosome-like vesicles. Cell. 2013;153(5):1120-33. 
27. Liu X, Jiang T, Li X, Zhao C, Li J, Zhou F, et al. Exosomes transmit T790M mutation-induced resistance in EGFR-mutant NSCLC by activating PI3K/AKT signalling pathway. Journal of cellular and molecular medicine. 2020;24(2):1529-40.

28. Sousa D, Lima RT, Vasconcelos MH. Intercellular Transfer of Cancer Drug Resistance Traits by Extracellular Vesicles. Trends in molecular medicine. 2015;21(10):595-608.

29. To KK. MicroRNA: a prognostic biomarker and a possible druggable target for circumventing multidrug resistance in cancer chemotherapy. Journal of biomedical science. 2013;20:99.

30. Koga Y, Yasunaga M, Moriya Y, Akasu T, Fujita S, Yamamoto S, et al. Exosome can prevent RNase from degrading microRNA in feces. Journal of gastrointestinal oncology. 2011;2(4):215-22.

31. Qin X, Yu S, Zhou L, Shi M, Hu Y, Xu X, et al. Cisplatin-resistant lung cancer cell-derived exosomes increase cisplatin resistance of recipient cells in exosomal miR-100-5p-dependent manner. International journal of nanomedicine. 2017;12:3721-33.

32. Zhang Y, Li M, Hu C. Exosomal transfer of miR-214 mediates gefitinib resistance in non-small cell lung cancer. Biochemical and biophysical research communications. 2018;507(1-4):457-64.

33. Valvona CJ, Fillmore HL, Nunn PB, Pilkington GJ. The Regulation and Function of Lactate Dehydrogenase A: Therapeutic Potential in Brain Tumor. Brain pathology (Zurich, Switzerland). 2016;26(1):3-17.

34. Zhang X, Guo M, Fan J, Lv Z, Huang Q, Han J, et al. Prognostic significance of serum LDH in small cell lung cancer: A systematic review with meta-analysis. Cancer biomarkers : section A of Disease markers. 2016;16(3):415-23.

35. He G, Jiang Z, Xue S, Sun X, Wang W. Expression of LDH and CEA in serum in the process of targeted therapy of lung adenocarcinoma and the association between them and prognosis. Oncology letters. 2019;17(5):4550-6.

36. Gao Y, Song P, Li H, Jia H, Zhang B. Elevated serum CEA levels are associated with the explosive progression of lung adenocarcinoma harboring EGFR mutations. BMC cancer. 2017;17(1):484.

37. Zhang H, Gao L, Zhang B, Zhang L, Wang C. Prognostic value of platelet to lymphocyte ratio in nonsmall cell lung cancer: a systematic review and meta-analysis. Scientific reports. 2016;6:22618.

38. Kim SH, Lee HW, Go SI, Lee SI, Lee GW. Clinical significance of the preoperative platelet count and platelet-to-lymphocyte ratio (PLT-PLR) in patients with surgically resected non-small cell lung cancer. Oncotarget. 2016;7(24):36198-206.

39. Huang YH, Hsu KH, Tseng JS, Chen KC, Hsu CH, Su KY, et al. The Association of Acquired T790M Mutation with Clinical Characteristics after Resistance to First-Line Epidermal Growth Factor Receptor Tyrosine Kinase Inhibitor in Lung Adenocarcinoma. Cancer research and treatment : official journal of Korean Cancer Association. 2018;50(4):1294-303.

40. Papadimitrakopoulou V, Wu YL, Han JY, Ahn MJ, Ramalingam S, John T, et al. LBA51Analysis of resistance mechanisms to osimertinib in patients with EGFR T790M advanced NSCLC from the AURA3 study. Annals of Oncology. 2018;29. 
41. Lin CC, Shih JY, Yu CJ, Ho CC, Liao WY, Lee JH, et al. Outcomes in patients with non-small-cell lung cancer and acquired Thr790Met mutation treated with osimertinib: a genomic study. The Lancet Respiratory medicine. 2018;6(2):107-16.

42. Oxnard GR, Hu Y, Mileham KF, Husain H, Costa DB, Tracy P, et al. Assessment of Resistance Mechanisms and Clinical Implications in Patients With EGFR T790M-Positive Lung Cancer and Acquired Resistance to Osimertinib. JAMA oncology. 2018;4(11):1527-34.

\section{Figures}
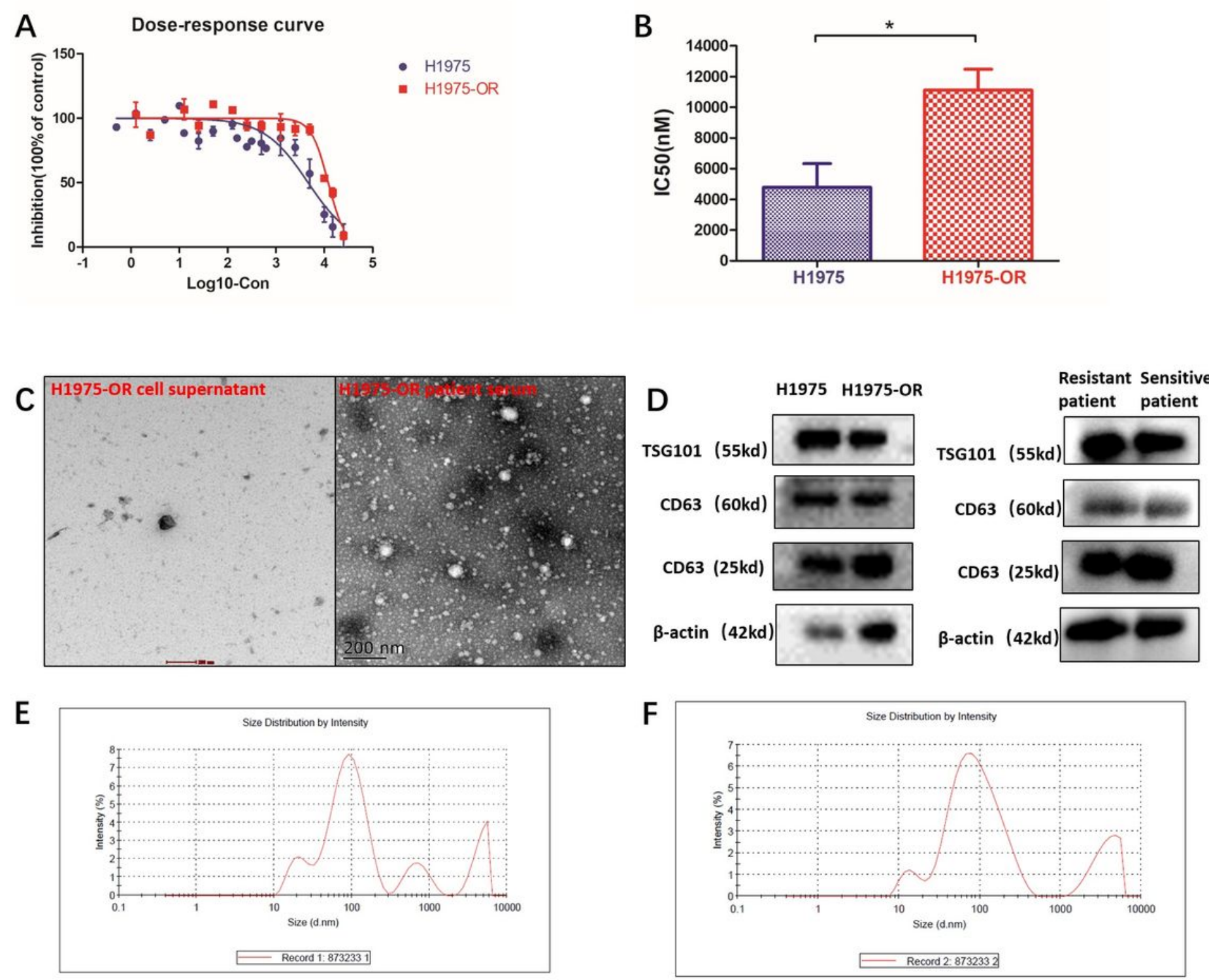

\section{Figure 1}

Isolation and identification of exosomes. (A) The drug-resistant strain, H1975-OR, was obtained by continuous exposure of $\mathrm{H} 1975$ to osimertinib for 6 months. After incubating H1975-OR and H1975 cells with different concentrations of osimertinib for $72 \mathrm{~h}$, the cell viability was measured using the MTT method. (B) The average IC50 values in H1975 and H1975-OR were 4636 nM and 12101 nM, respectively, 
and the difference was statistically significant $(p=0.0215)$. (C) The exosomes $(30-150 \mathrm{~nm})$ extracted from the H1975-OR cell supernatant and the osimertinib-resistant patient were observed using electron microscopy. Scale $=200 \mathrm{~nm}$. (D) Exosomes were identified using western blot. (E) and (F) particle size analysis of exosomes in the cell supernatant and plasma of patients, respectively.
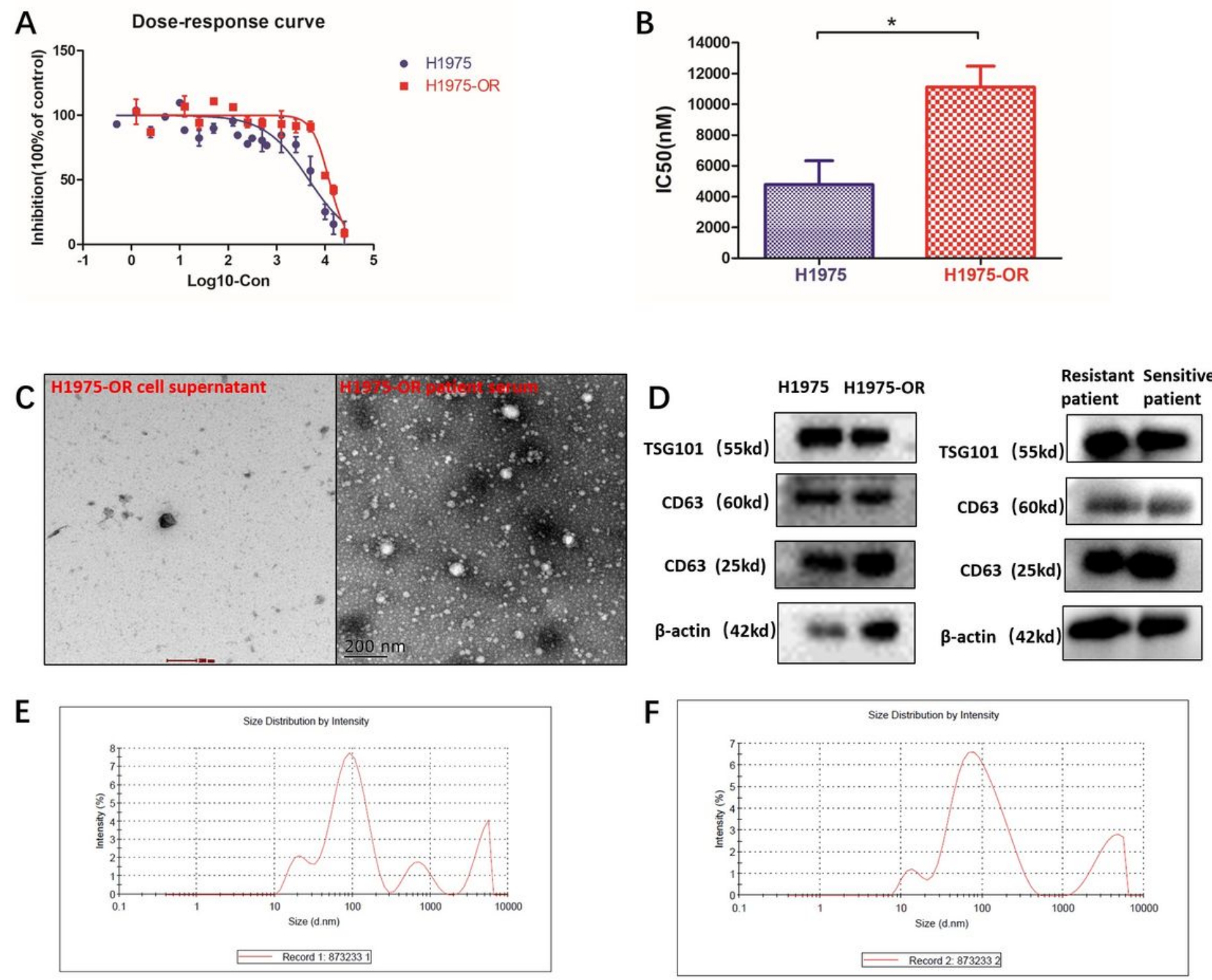

\section{Figure 1}

Isolation and identification of exosomes. (A) The drug-resistant strain, H1975-OR, was obtained by continuous exposure of $\mathrm{H} 1975$ to osimertinib for 6 months. After incubating H1975-OR and H1975 cells with different concentrations of osimertinib for $72 \mathrm{~h}$, the cell viability was measured using the MTT method. (B) The average IC50 values in H1975 and H1975-OR were $4636 \mathrm{nM}$ and $12101 \mathrm{nM}$, respectively, and the difference was statistically significant $(p=0.0215)$. (C) The exosomes $(30-150 \mathrm{~nm})$ extracted from the H1975-OR cell supernatant and the osimertinib-resistant patient were observed using electron microscopy. Scale $=200 \mathrm{~nm}$. (D) Exosomes were identified using western blot. (E) and (F) particle size analysis of exosomes in the cell supernatant and plasma of patients, respectively. 
A

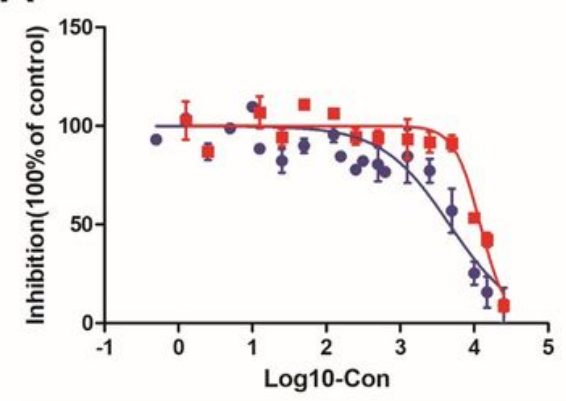

C

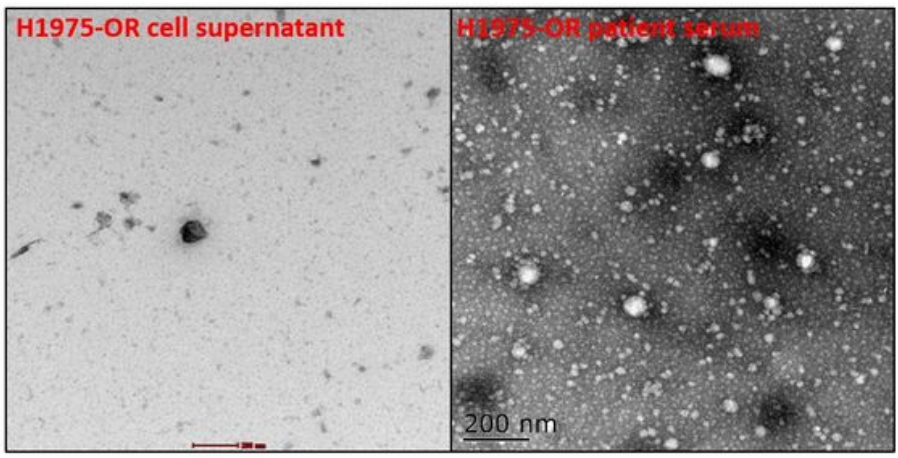

E

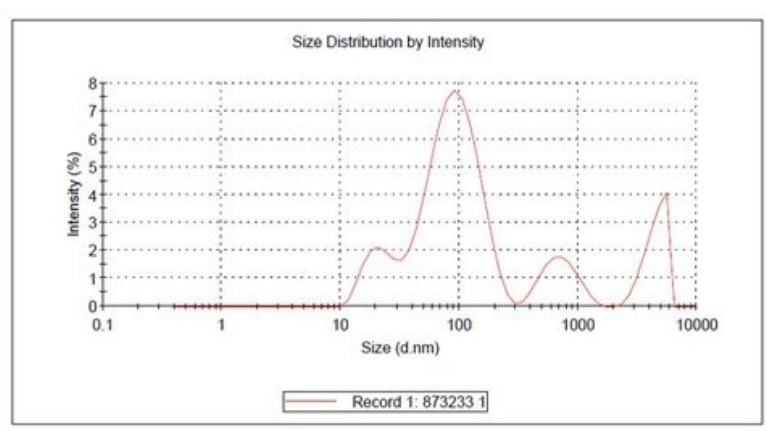

B

- $\mathrm{H} 1975$

- H1975-OR
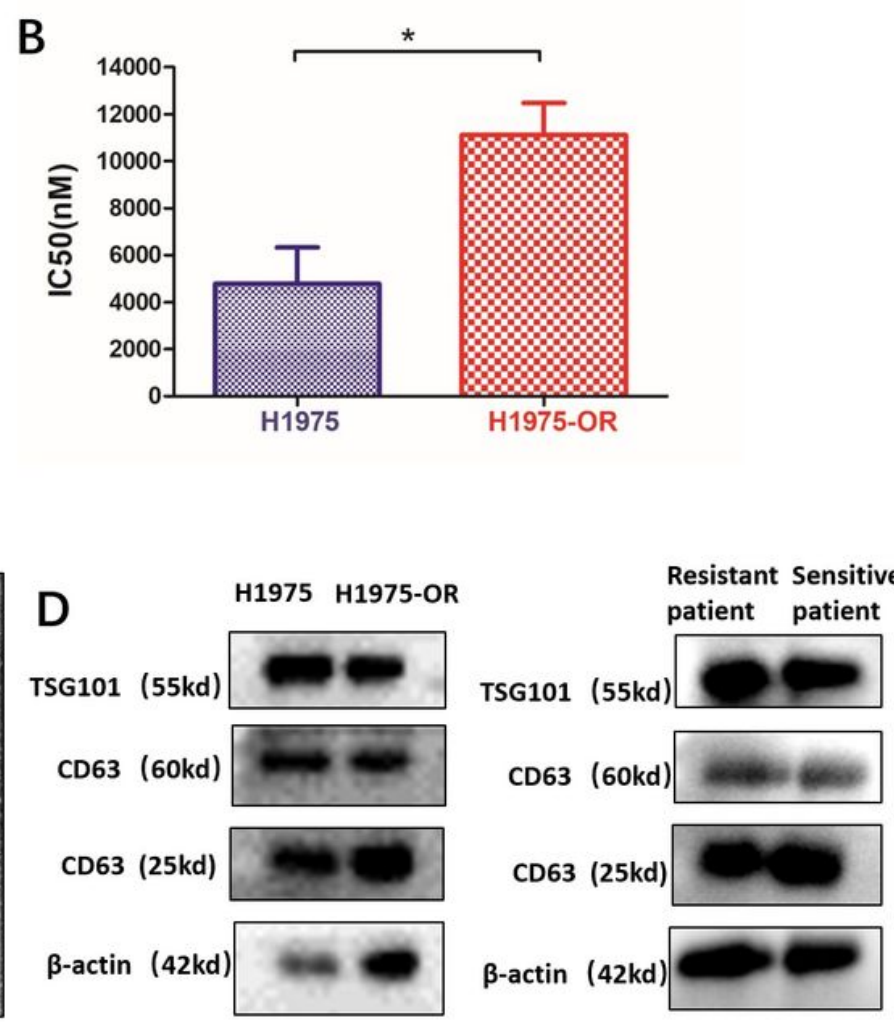

$\mathrm{F}$

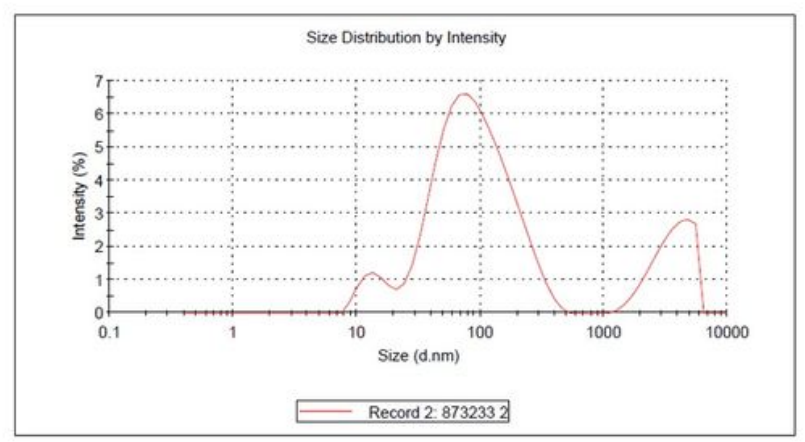

Figure 1

Isolation and identification of exosomes. (A) The drug-resistant strain, H1975-OR, was obtained by continuous exposure of H1975 to osimertinib for 6 months. After incubating H1975-OR and H1975 cells with different concentrations of osimertinib for $72 \mathrm{~h}$, the cell viability was measured using the MTT method. (B) The average IC50 values in H1975 and H1975-OR were $4636 \mathrm{nM}$ and $12101 \mathrm{nM}$, respectively, and the difference was statistically significant $(p=0.0215)$. (C) The exosomes $(30-150 \mathrm{~nm})$ extracted from the H1975-OR cell supernatant and the osimertinib-resistant patient were observed using electron microscopy. Scale $=200 \mathrm{~nm}$. (D) Exosomes were identified using western blot. (E) and (F) particle size analysis of exosomes in the cell supernatant and plasma of patients, respectively. 
A

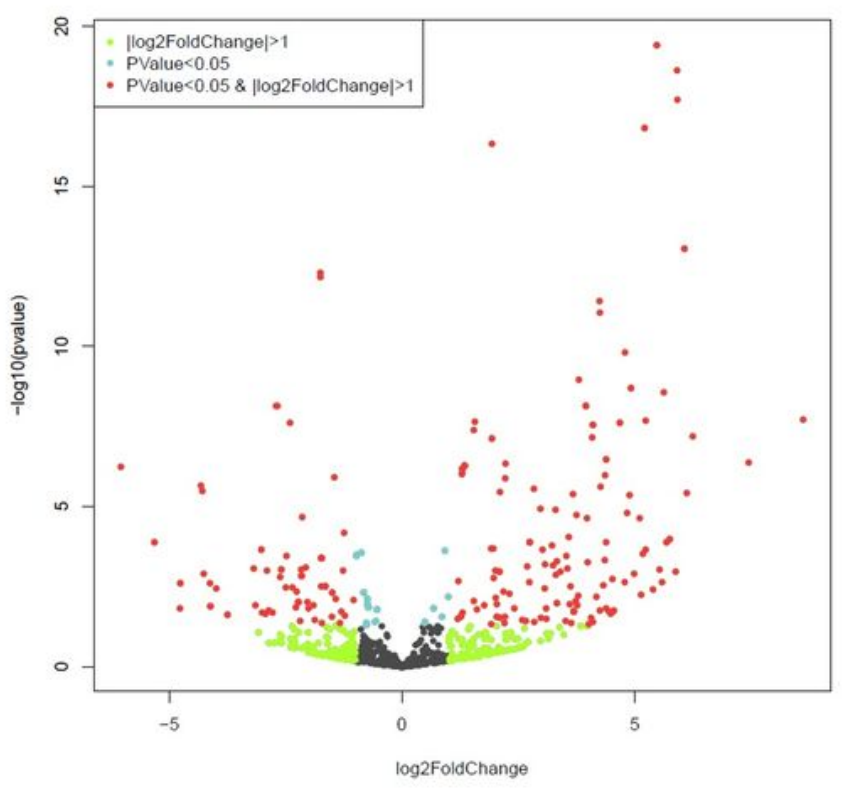

C

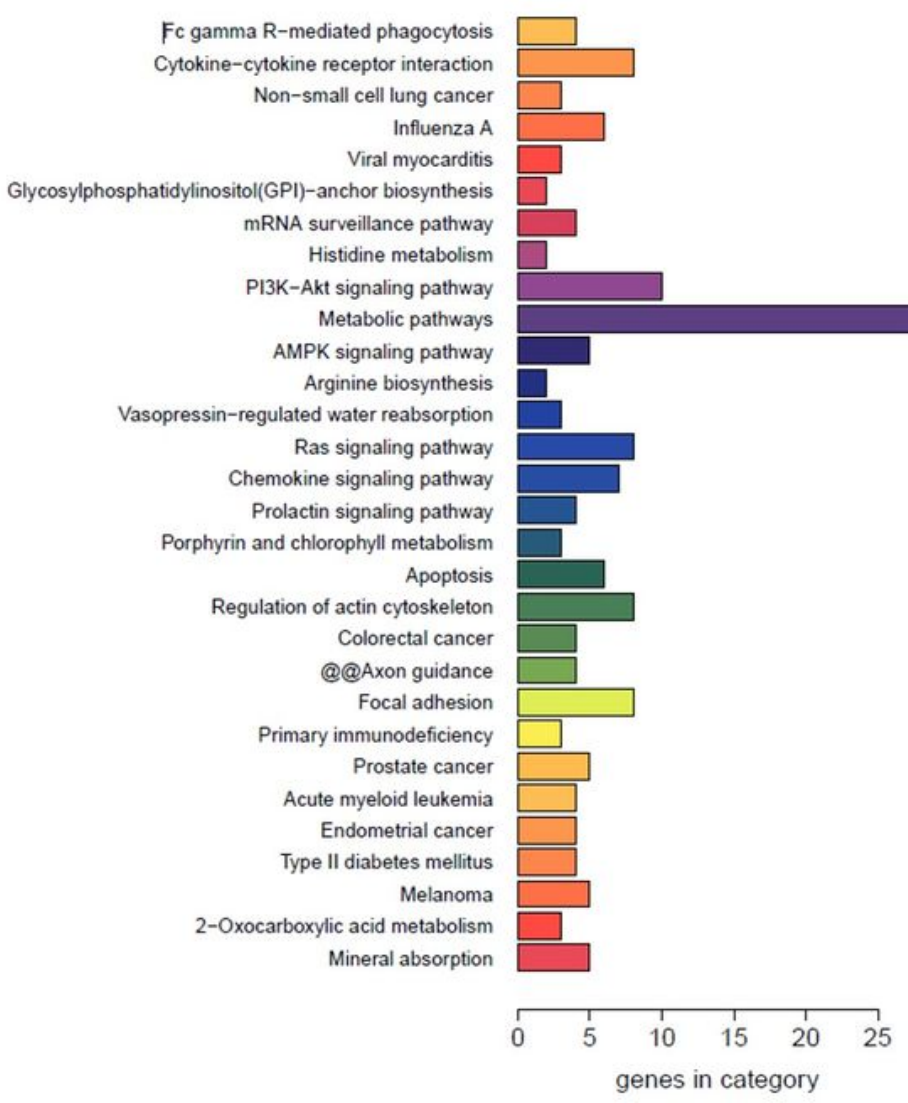

B

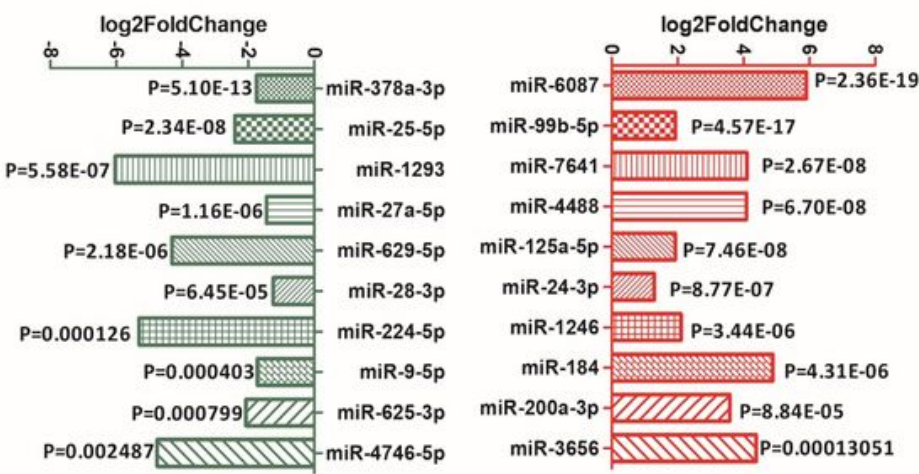

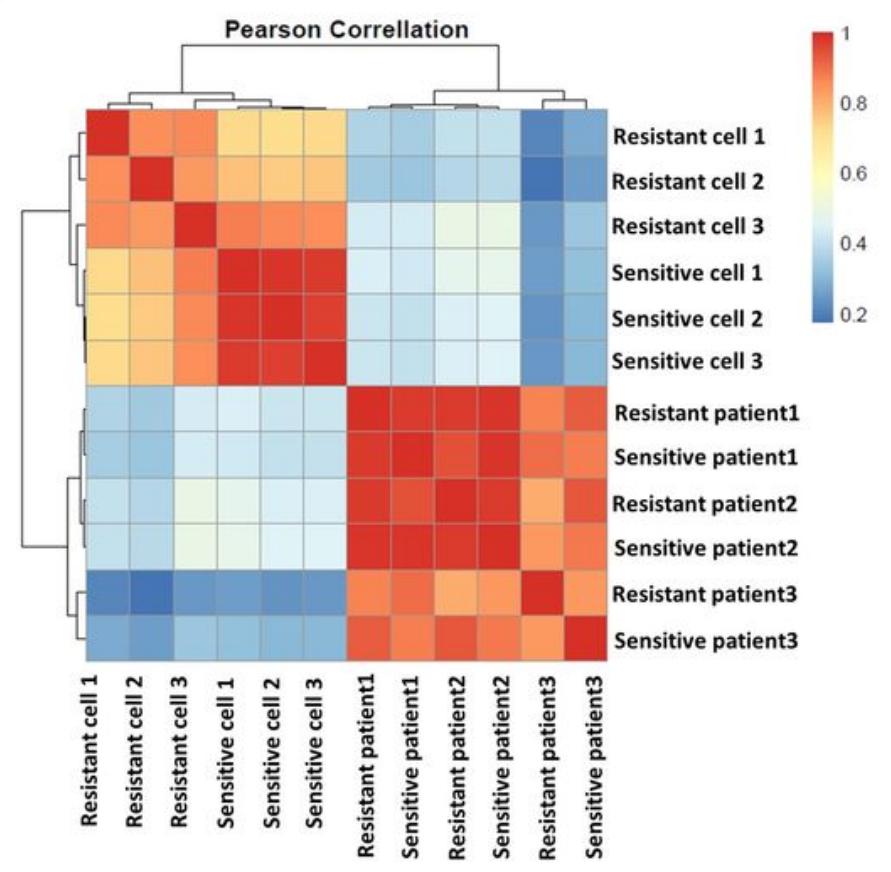

Figure 2

Cluster analysis of miRNAs and target genes. (A) Volcano map of differentially expressed miRNAs. Each point in the volcano map represents a gene, and the red dots are significantly different genes that satisfy both $[\log F C]>1$ and FDR $<0.05$ thresholds. The blue dots are genes that only meet FDR $<0.05$, and the green dots are genes that only meet [logFC]> 1. (B) H1975-OR top 10 up-regulated miRNAs (red) and top 10 down-regulated miRNAs (green) compared with H1975. (C) KEGG pathway enrichment analysis of 
target genes for differentially expressed miRNA (histogram). The ordinate represents the name of the pathway, and the abscissa is the number of genes involved in each pathway. (D) Correlation analysis of exosome miRNA in patient serum and the $\mathrm{H} 1975$ cell supernatant. The correlation coefficient uses Pearson's correlation test (column, row), and the color of each square corresponds to the correlation coefficient value.

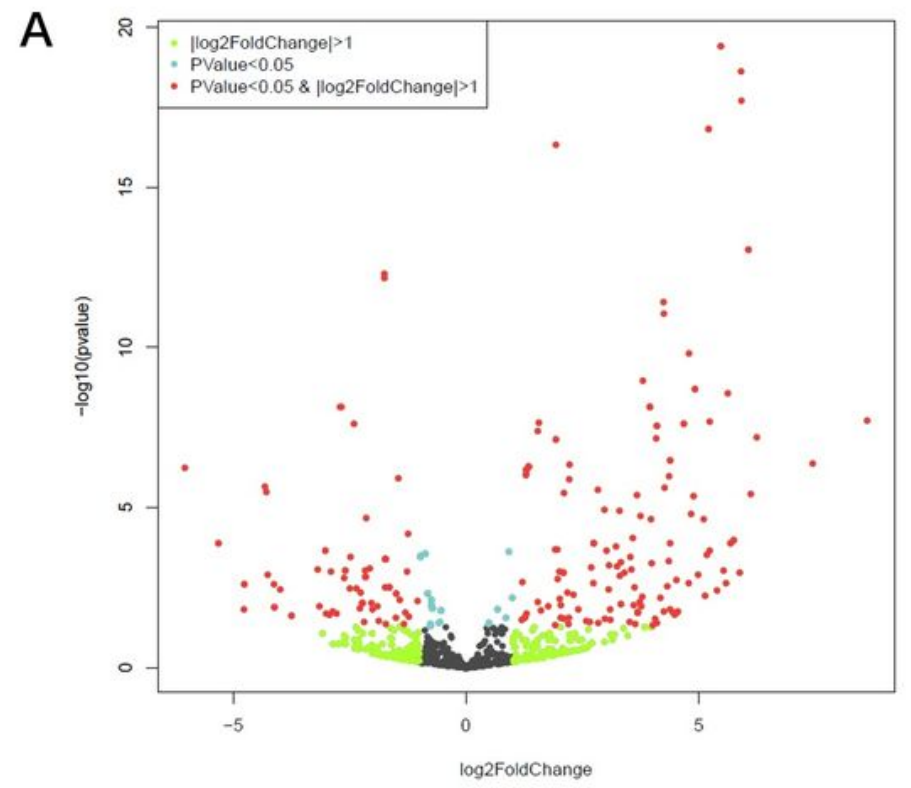

B
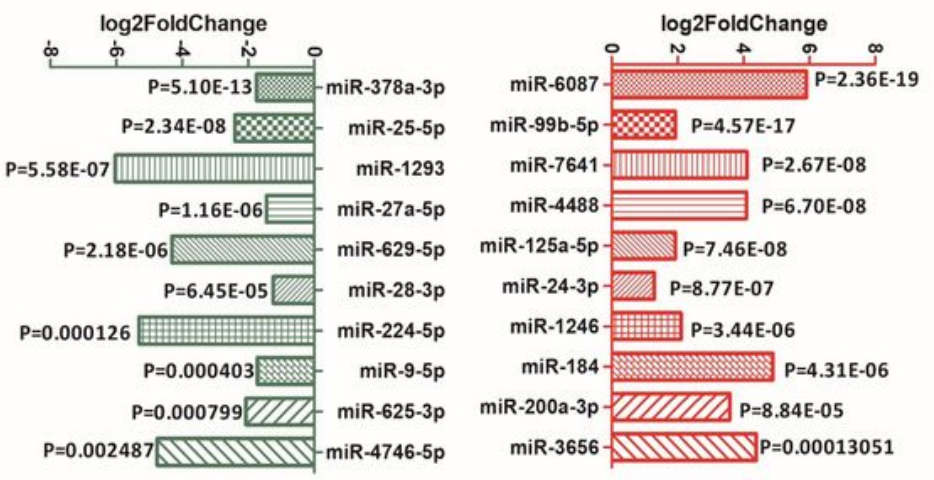

C

KEGG Pathway Enrichment
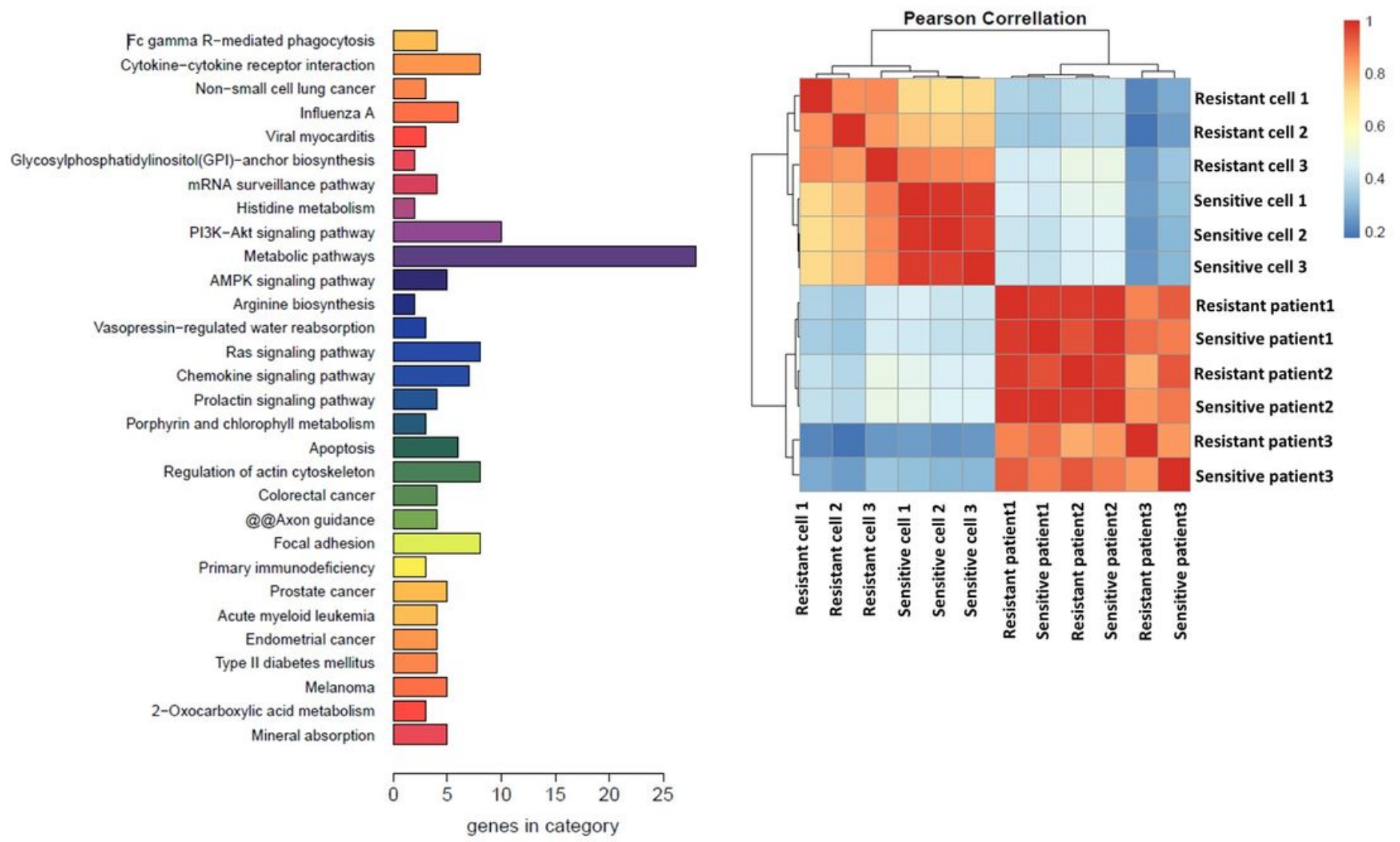

Figure 2 
Cluster analysis of miRNAs and target genes. (A) Volcano map of differentially expressed miRNAs. Each point in the volcano map represents a gene, and the red dots are significantly different genes that satisfy both [logFC] $>1$ and FDR $<0.05$ thresholds. The blue dots are genes that only meet FDR $<0.05$, and the green dots are genes that only meet [logFC]> 1. (B) H1975-OR top 10 up-regulated miRNAs (red) and top 10 down-regulated miRNAs (green) compared with H1975. (C) KEGG pathway enrichment analysis of target genes for differentially expressed miRNA (histogram). The ordinate represents the name of the pathway, and the abscissa is the number of genes involved in each pathway. (D) Correlation analysis of exosome miRNA in patient serum and the $\mathrm{H} 1975$ cell supernatant. The correlation coefficient uses Pearson's correlation test (column, row), and the color of each square corresponds to the correlation coefficient value. 
A

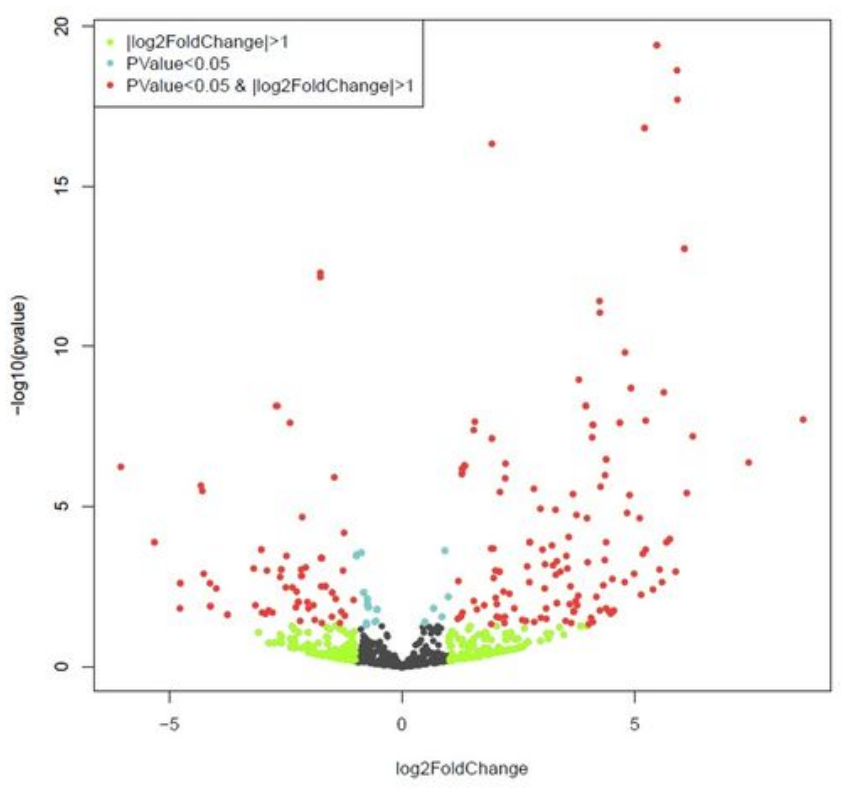

C

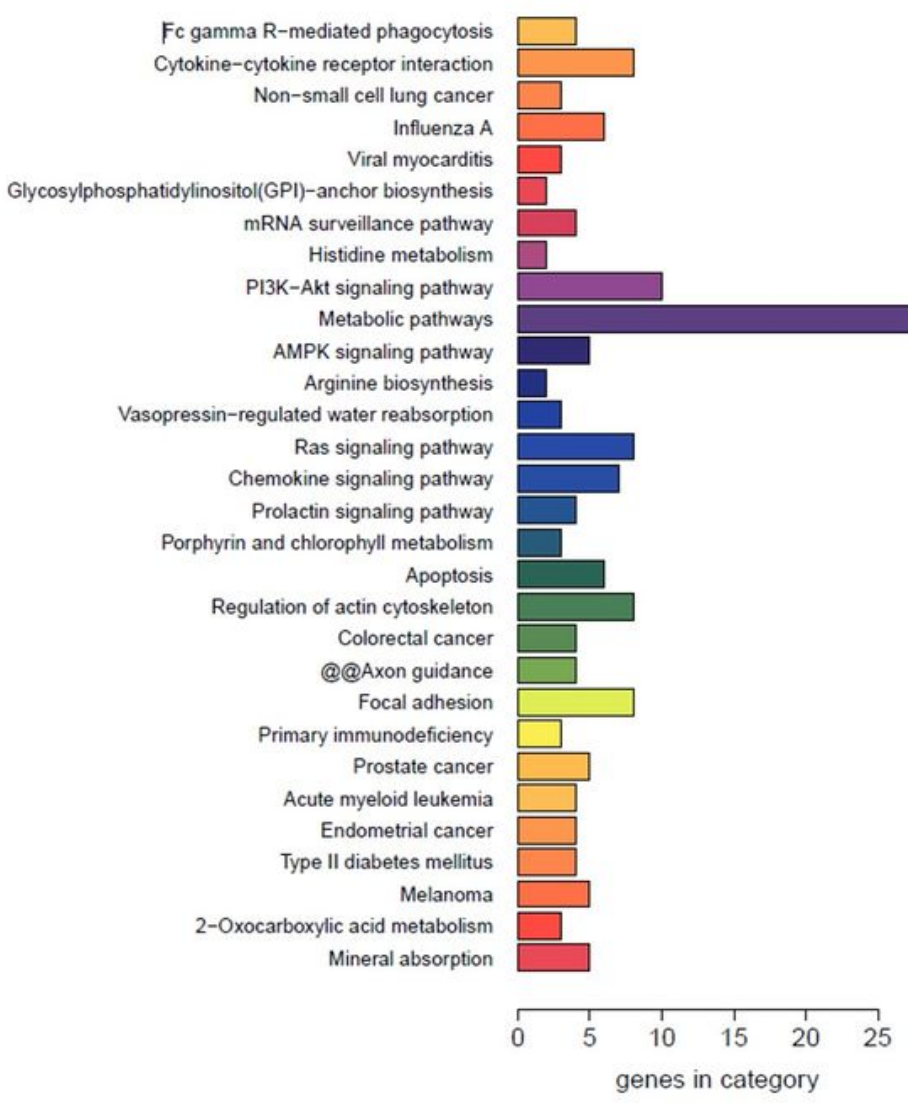

B

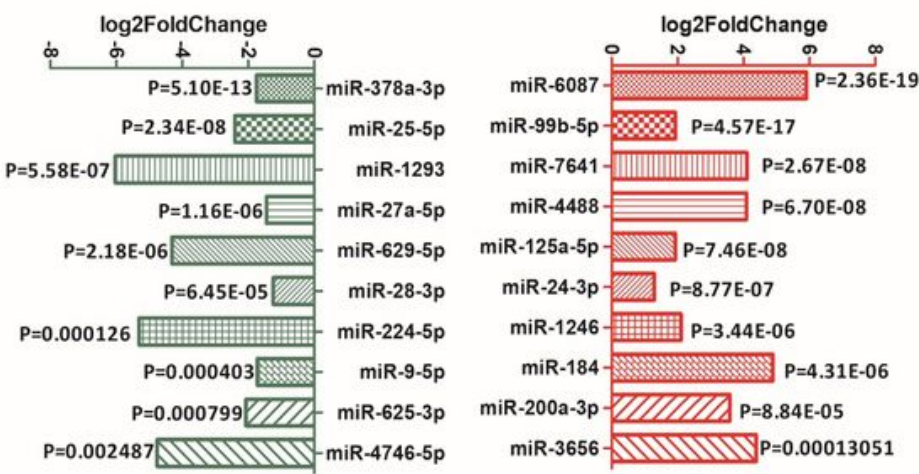

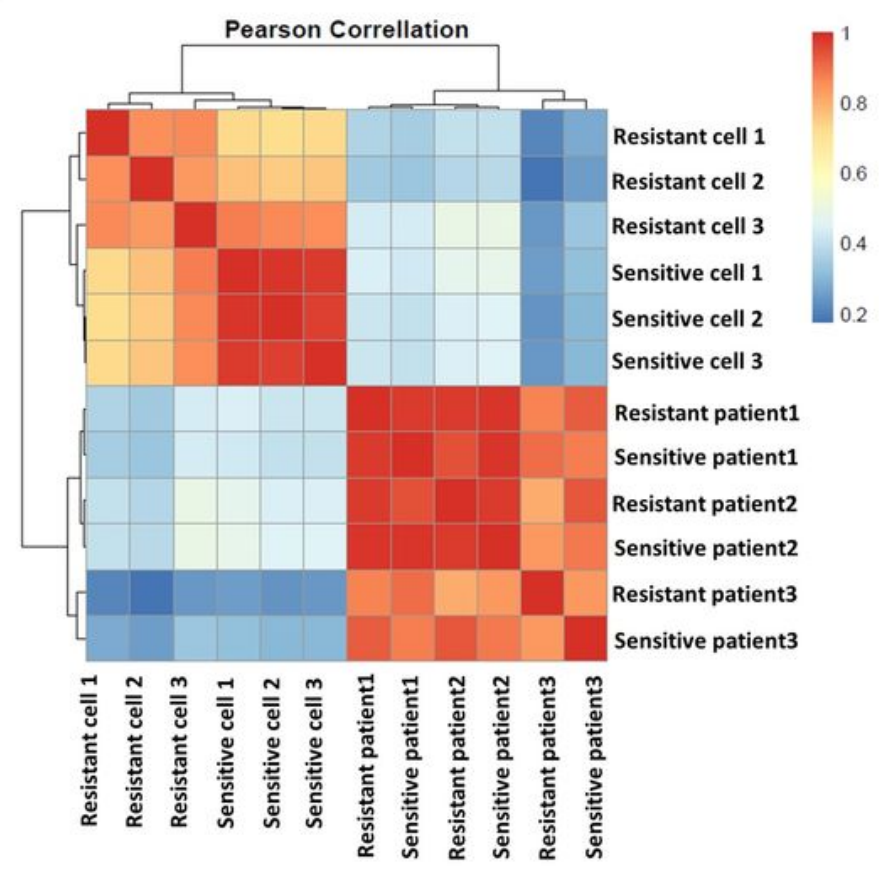

Figure 2

Cluster analysis of miRNAs and target genes. (A) Volcano map of differentially expressed miRNAs. Each point in the volcano map represents a gene, and the red dots are significantly different genes that satisfy both $[\log F C]>1$ and FDR $<0.05$ thresholds. The blue dots are genes that only meet FDR $<0.05$, and the green dots are genes that only meet [logFC]> 1. (B) H1975-OR top 10 up-regulated miRNAs (red) and top 10 down-regulated miRNAs (green) compared with H1975. (C) KEGG pathway enrichment analysis of 
target genes for differentially expressed miRNA (histogram). The ordinate represents the name of the pathway, and the abscissa is the number of genes involved in each pathway. (D) Correlation analysis of exosome miRNA in patient serum and the $\mathrm{H} 1975$ cell supernatant. The correlation coefficient uses Pearson's correlation test (column, row), and the color of each square corresponds to the correlation coefficient value.

A

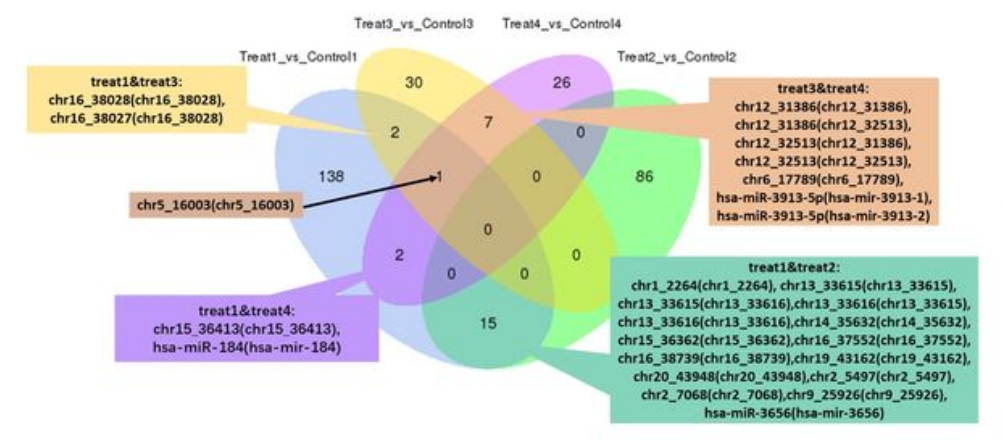

C

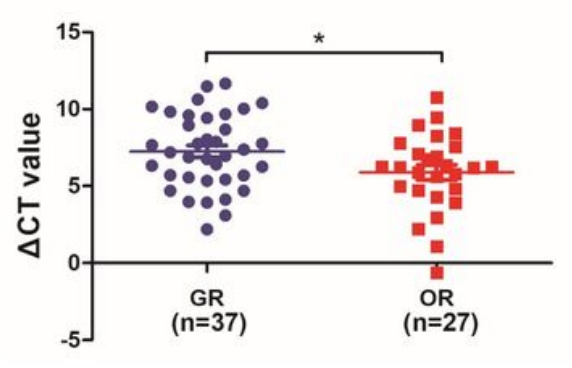

F ROC curve

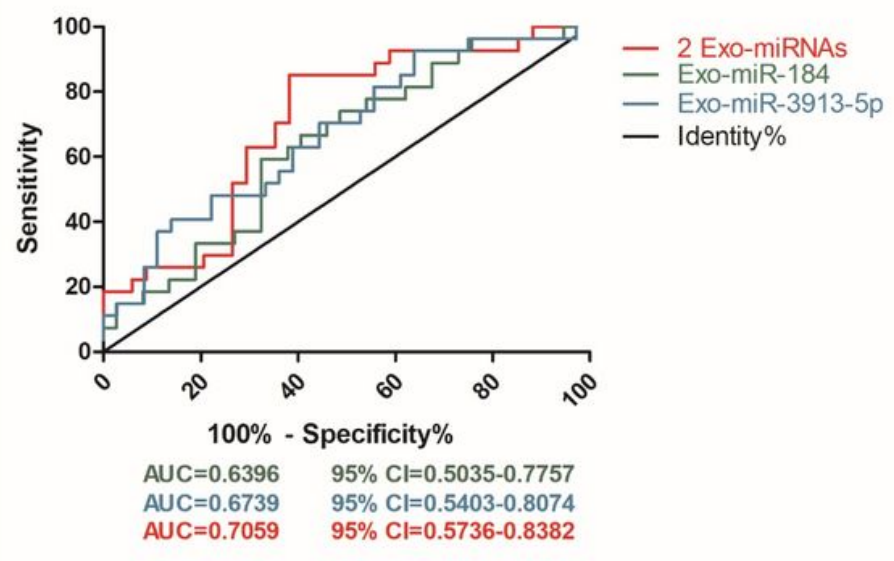

B

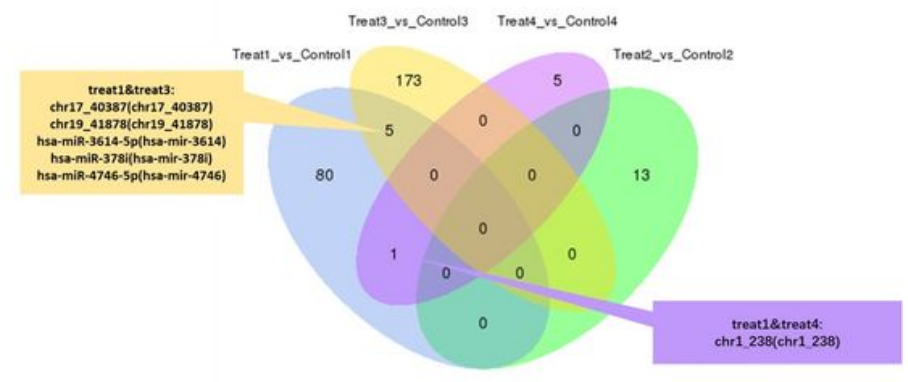

D

Exo-miR3913-5p

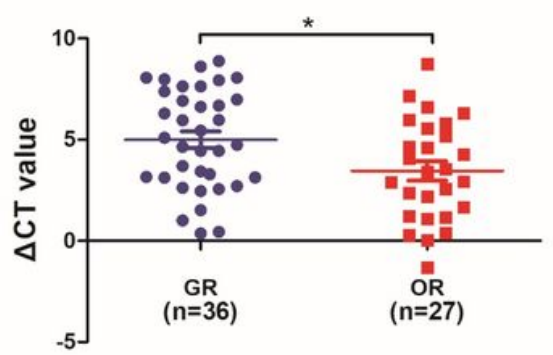

$\mathrm{E}$

EXO-miRNAs

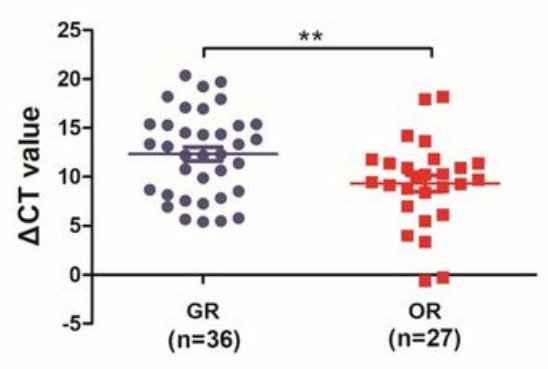

\section{Figure 3}

MiR-184 and miR-3913-5p elevated expression in exosomes after osimertinib resistance. (A) Overlaid analysis of Wayne maps of exosomal miRNAs up-regulated and (B) down-regulated exosomal miRNAs in the drug-resistant group compared to that in the sensitive group. (C) q-PCR exosome miR-184 expression levels in patients with osimertinib-resistant NSCLC $(O R, n=27)$ and patients without resistance $(G R, n=$ 37), * $p<0.05$. (D) Serum exosomal miR-3913-5p expression levels in patients with osimertinib-resistant 
NSCLC (OR, $n=27)$ and osimertinib-sensitive patients ( $G R, n=36)$, * $p<0.05$. (E) Changes in the expression levels of the two exosomal miRNAs before and after resistance to osimertinib, ${ }^{\star *} p<0.01$. (F) ROC curves for miR-184 (green), miR-3913-5p (blue), and the two miRNAs (red), respectively.

A

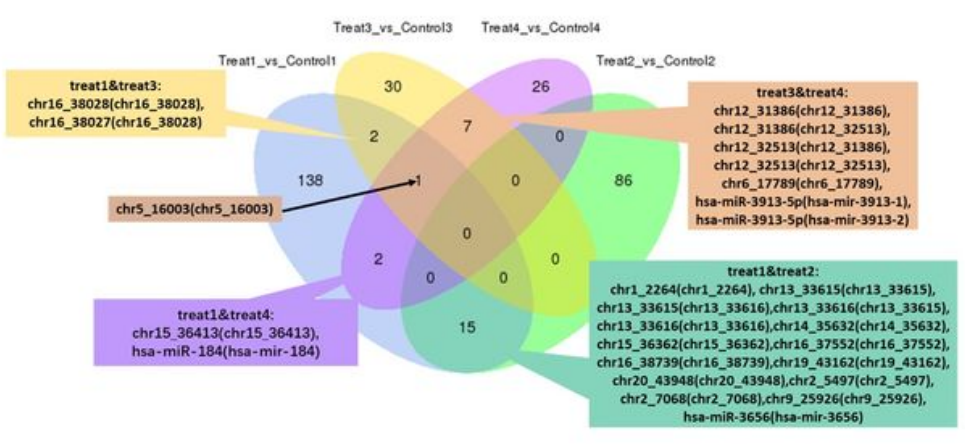

B

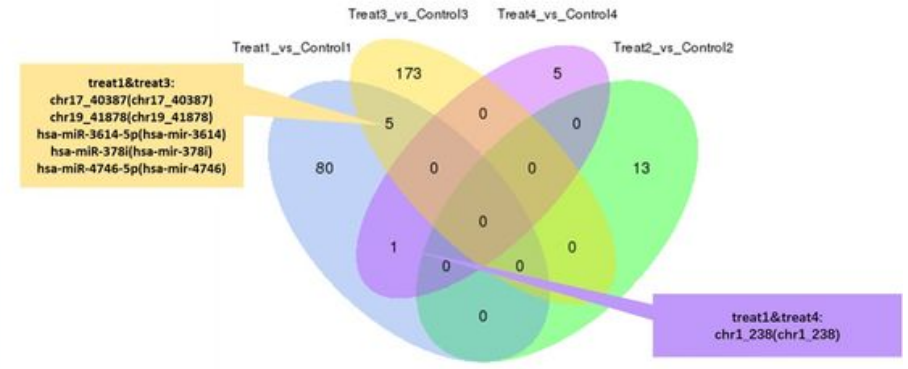

C

Exo-miR184

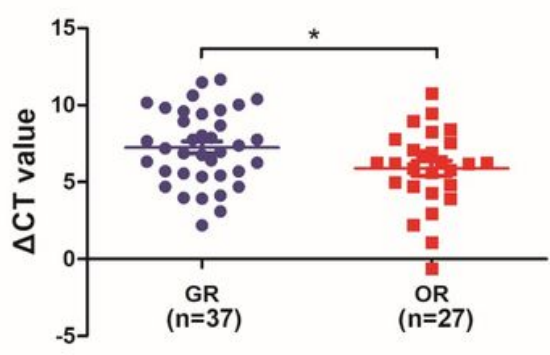

F ROC curve

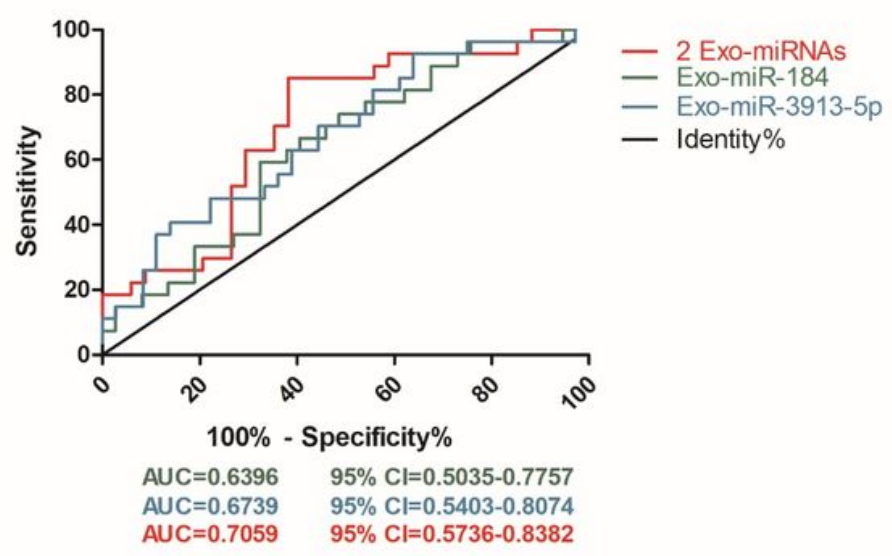

Exo-miR3913-5p

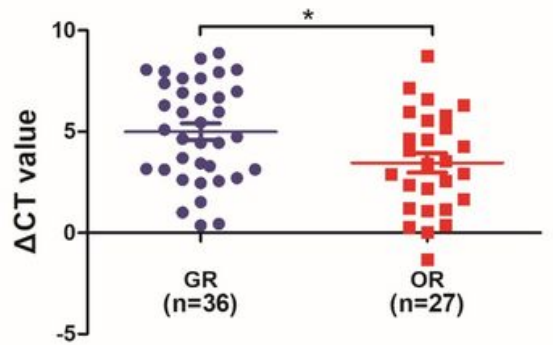

$\mathrm{E}$

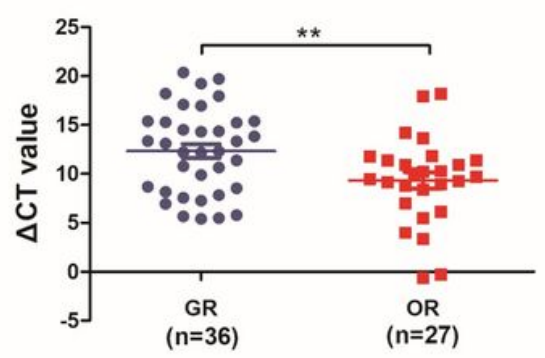

Figure 3

MiR-184 and miR-3913-5p elevated expression in exosomes after osimertinib resistance. (A) Overlaid analysis of Wayne maps of exosomal miRNAs up-regulated and (B) down-regulated exosomal miRNAs in the drug-resistant group compared to that in the sensitive group. (C) q-PCR exosome miR-184 expression levels in patients with osimertinib-resistant $\operatorname{NSCLC~}(O R, n=27)$ and patients without resistance $(G R, n=$ 37), * $p<0.05$. (D) Serum exosomal miR-3913-5p expression levels in patients with osimertinib-resistant NSCLC (OR, $n=27)$ and osimertinib-sensitive patients $(G R, n=36)$, * $p<0.05$. (E) Changes in the 
expression levels of the two exosomal miRNAs before and after resistance to osimertinib, ${ }^{\star \star} \mathrm{p}<0.01$. (F) ROC curves for miR-184 (green), miR-3913-5p (blue), and the two miRNAs (red), respectively.

A

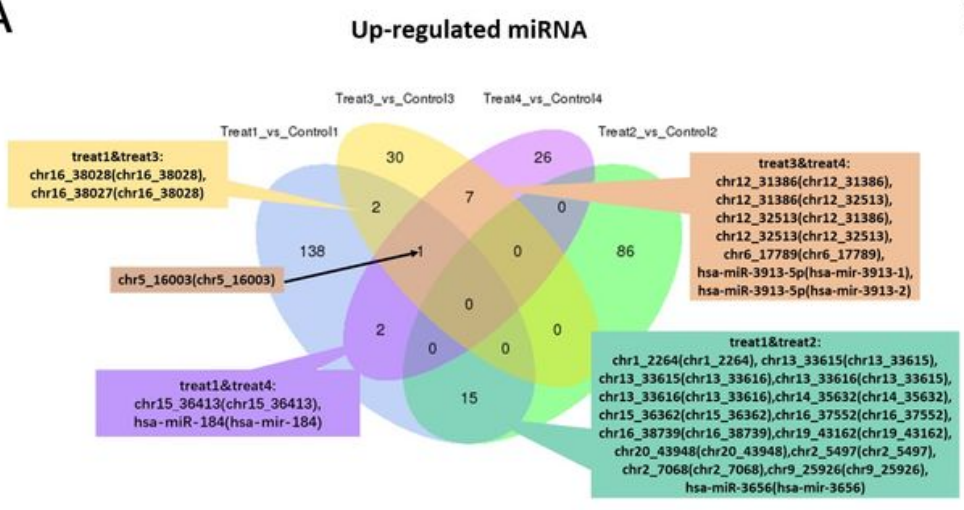

C

Exo-miR184

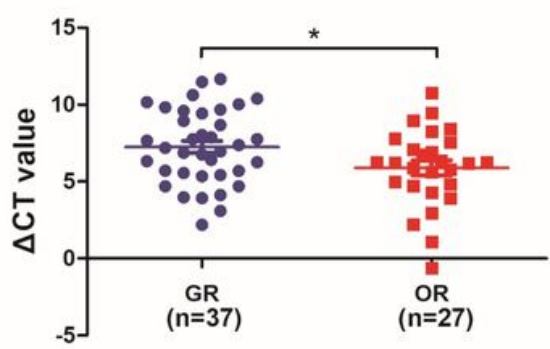

$\mathbf{F}$

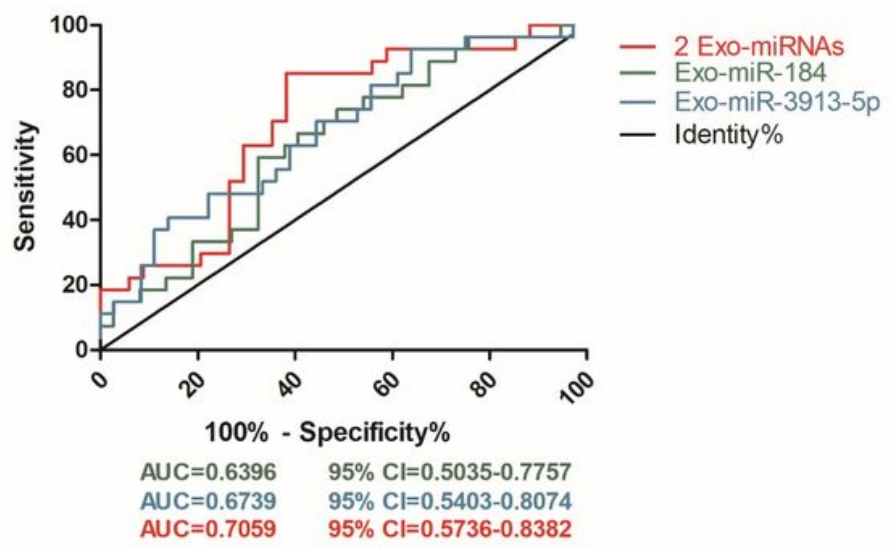

B

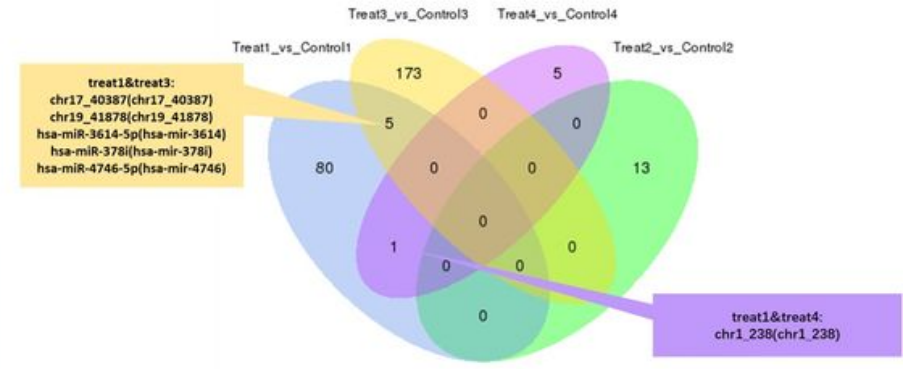

$\mathrm{E}$

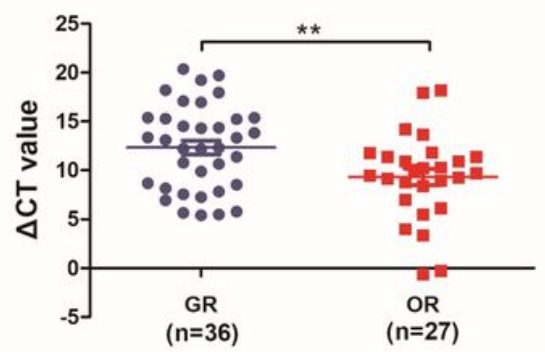

Figure 3

MiR-184 and miR-3913-5p elevated expression in exosomes after osimertinib resistance. (A) Overlaid analysis of Wayne maps of exosomal miRNAs up-regulated and (B) down-regulated exosomal miRNAs in the drug-resistant group compared to that in the sensitive group. (C) q-PCR exosome miR-184 expression levels in patients with osimertinib-resistant $\operatorname{NSCLC~}(O R, n=27)$ and patients without resistance $(G R, n=$ 37), * $p<0.05$. (D) Serum exosomal miR-3913-5p expression levels in patients with osimertinib-resistant NSCLC $(O R, n=27)$ and osimertinib-sensitive patients $(G R, n=36)$, * $p<0.05$. (E) Changes in the expression levels of the two exosomal miRNAs before and after resistance to osimertinib, ${ }^{\star \star} p<0.01$. (F) ROC curves for miR-184 (green), miR-3913-5p (blue), and the two miRNAs (red), respectively. 
A

EGFR 21 L858R

Exo-miR-184

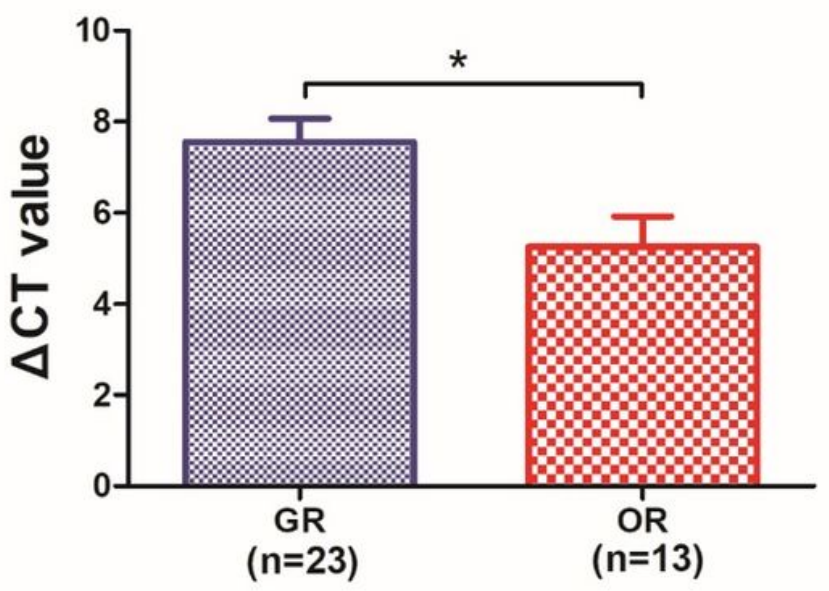

C

T790M $^{+}$

Exo-miR-184

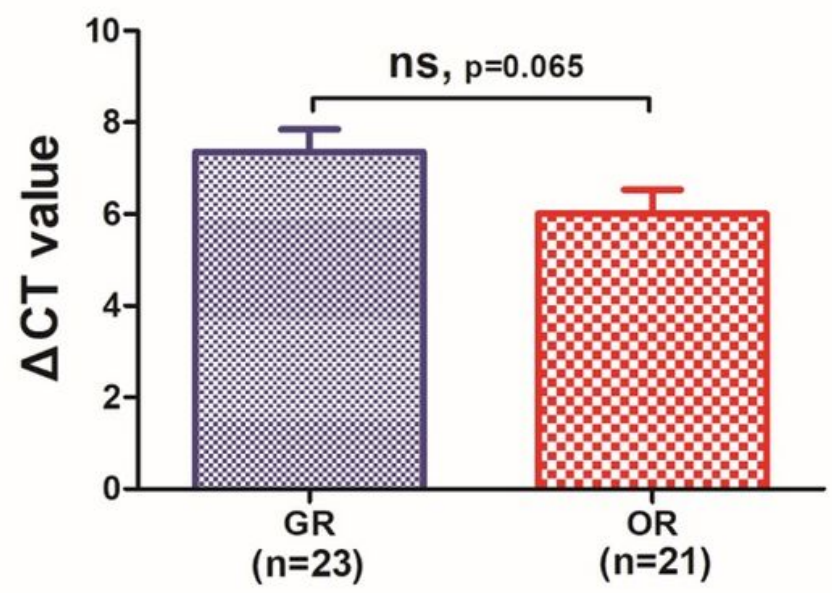

B

EGFR 21 L858R

Exo-miR-3913-5p

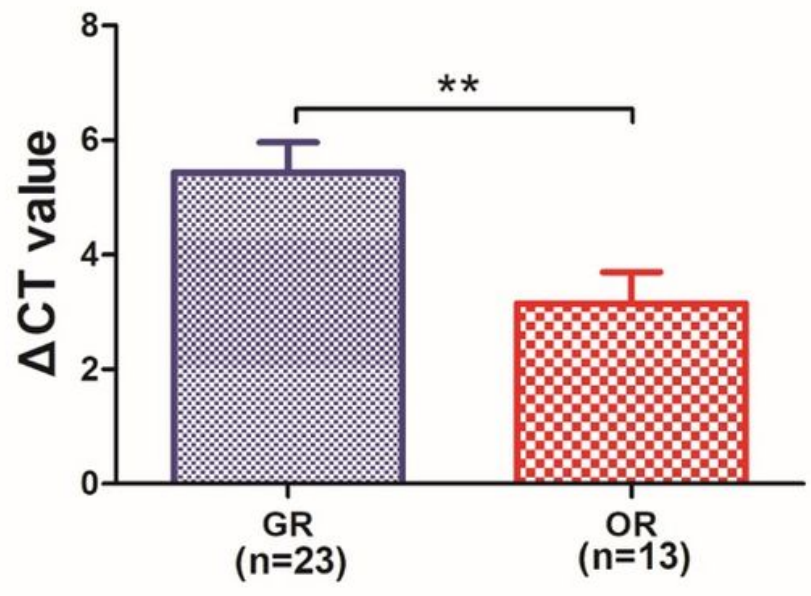

D T790M $^{+}$

Exo-miR-3913-5p

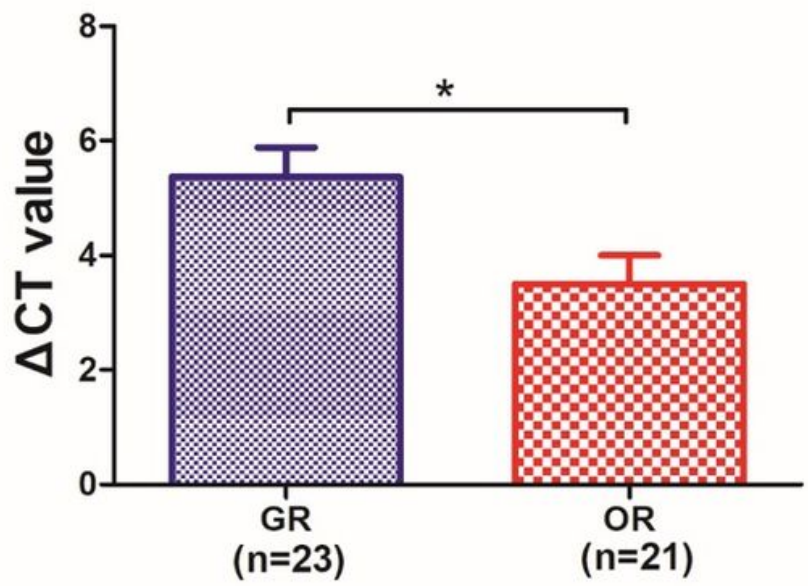

Figure 4

Osimertinib resistance induced by exosomal miRNAs targeting EGFR 21 mutations and T790M + patients. (A) q-PCR analysis of serum exosomal miR-184 and (B) miR-3913-5p expression levels in patients with drug resistance and sensitivity, in all patients with EGFR 19 exon deletion. (C) q-PCR analysis of serum exosomal miR-184 and (D) miR-3913-5p expression levels in patients with resistance and sensitivity in all patients with EGFR 19 exon L858R mutation. 
A

EGFR 21 L858R

Exo-miR-184

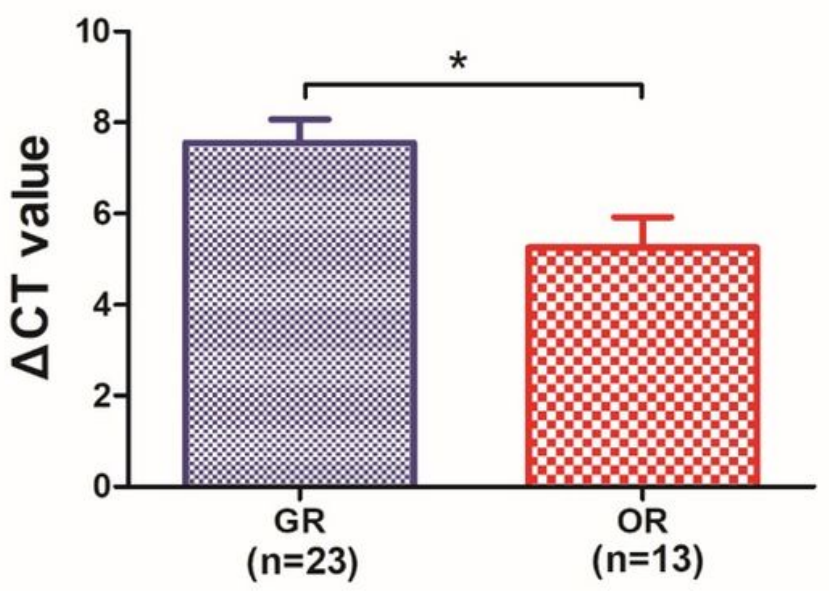

C

${\mathrm{T} 790 \mathrm{M}^{+}}^{+}$

Exo-miR-184

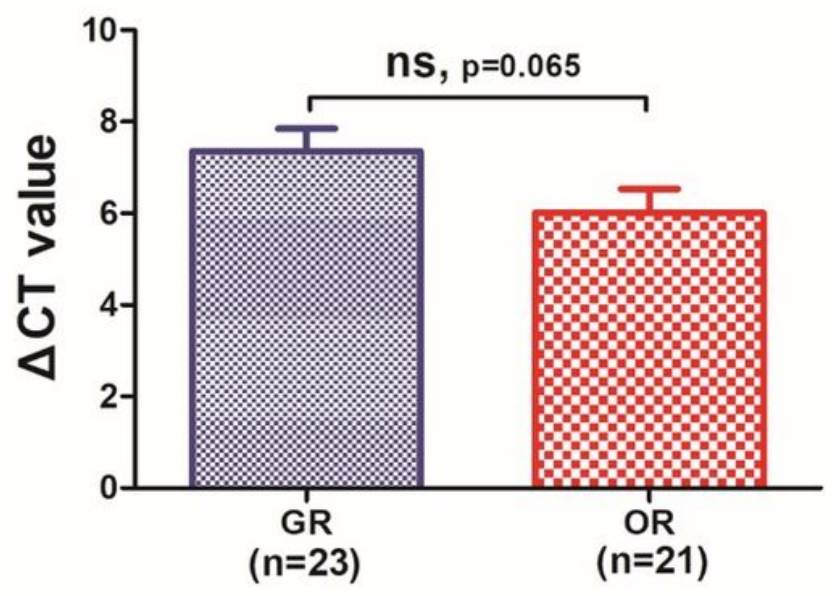

B

EGFR 21 L858R

Exo-miR-3913-5p

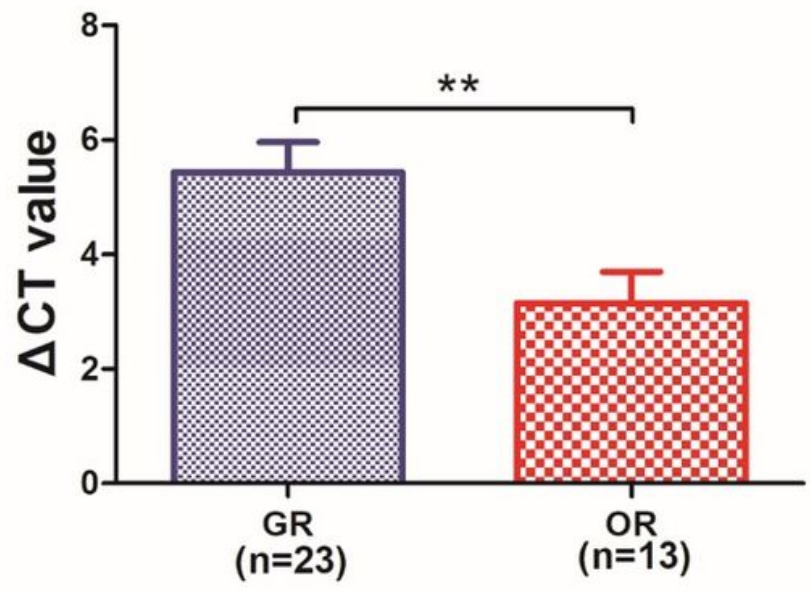

D $\mathrm{T}^{2} \mathrm{M}^{+}$

Exo-miR-3913-5p

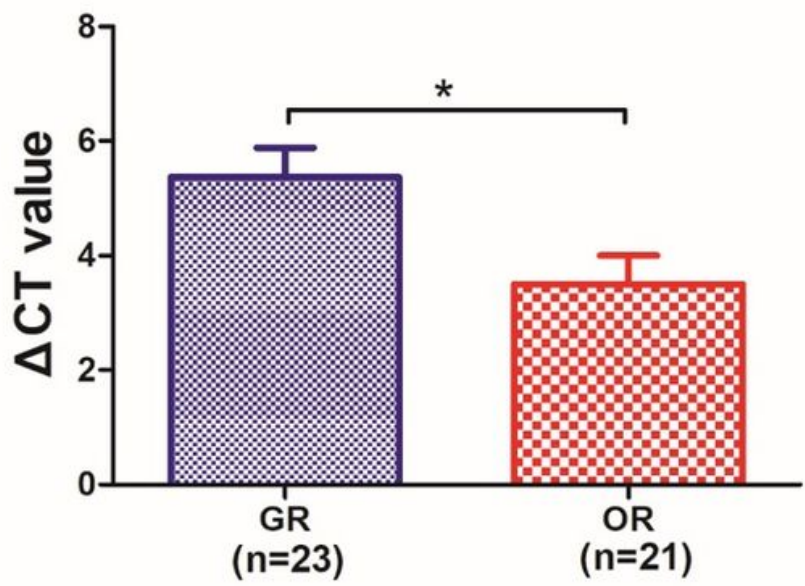

Figure 4

Osimertinib resistance induced by exosomal miRNAs targeting EGFR 21 mutations and T790M + patients. (A) q-PCR analysis of serum exosomal miR-184 and (B) miR-3913-5p expression levels in patients with drug resistance and sensitivity, in all patients with EGFR 19 exon deletion. (C) q-PCR analysis of serum exosomal miR-184 and (D) miR-3913-5p expression levels in patients with resistance and sensitivity in all patients with EGFR 19 exon L858R mutation. 
A

EGFR 21 L858R

Exo-miR-184

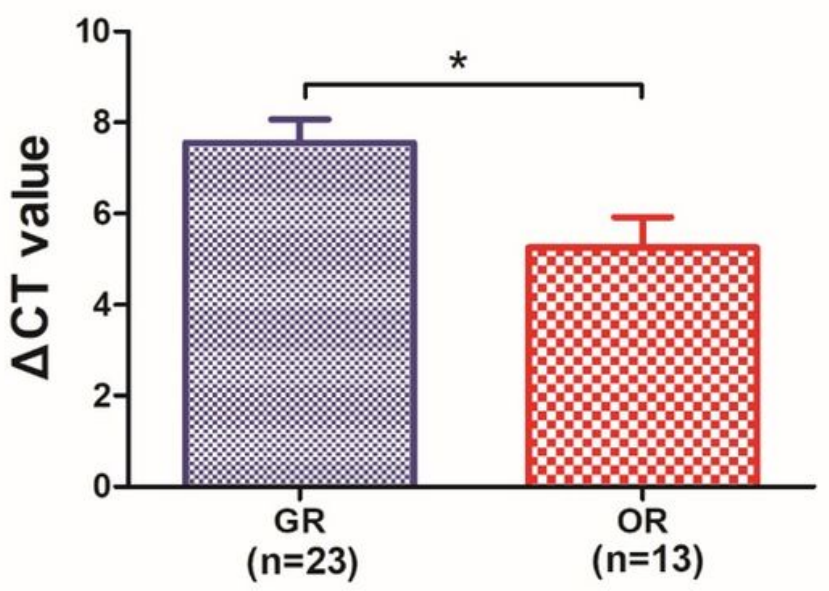

C

${\mathrm{T} 790 \mathrm{M}^{+}}^{+}$

Exo-miR-184

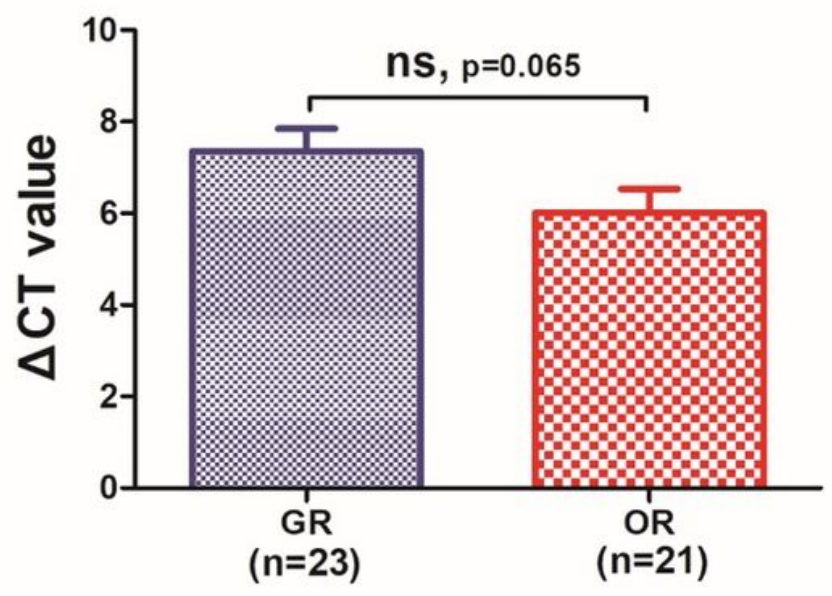

B

EGFR 21 L858R

Exo-miR-3913-5p

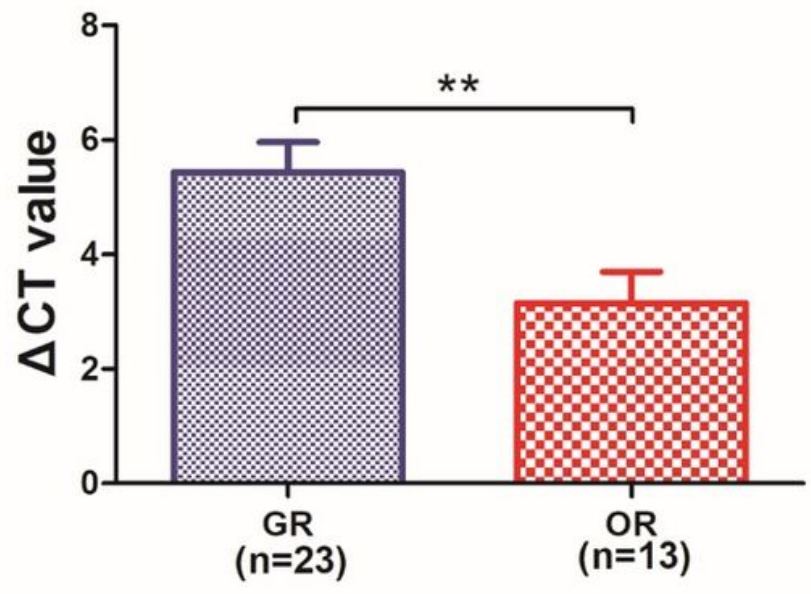

D $\mathrm{T}^{2} \mathrm{M}^{+}$

Exo-miR-3913-5p

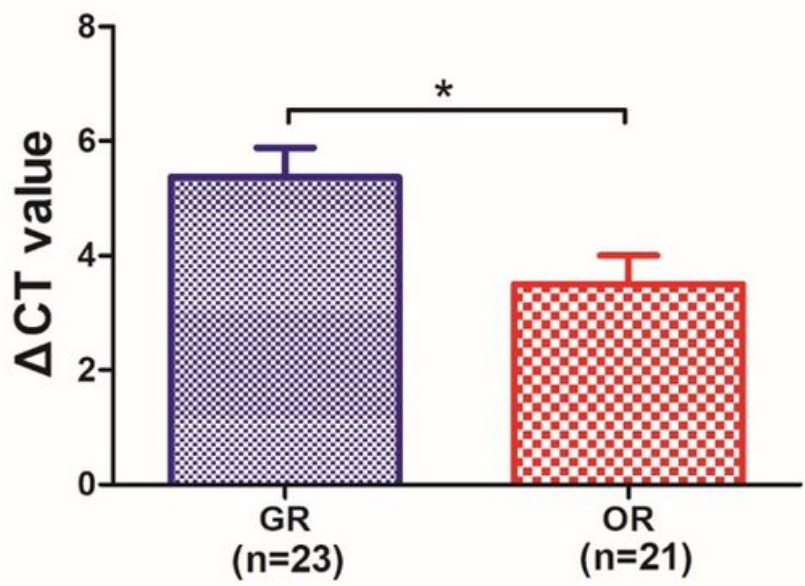

Figure 4

Osimertinib resistance induced by exosomal miRNAs targeting EGFR 21 mutations and T790M + patients. (A) q-PCR analysis of serum exosomal miR-184 and (B) miR-3913-5p expression levels in patients with drug resistance and sensitivity, in all patients with EGFR 19 exon deletion. (C) q-PCR analysis of serum exosomal miR-184 and (D) miR-3913-5p expression levels in patients with resistance and sensitivity in all patients with EGFR 19 exon L858R mutation. 

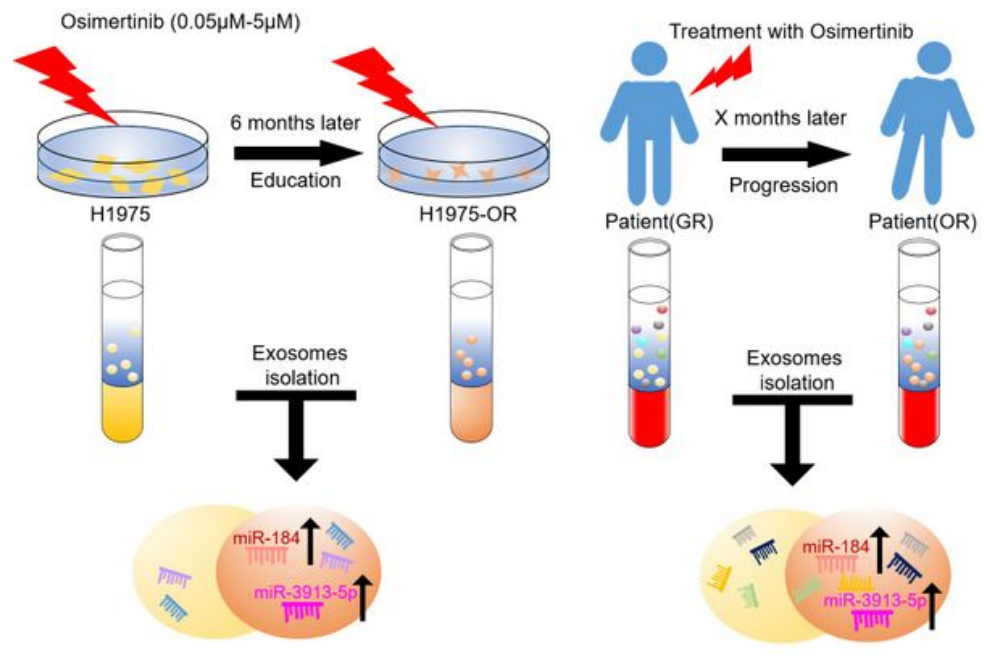

Bypass pathways activation

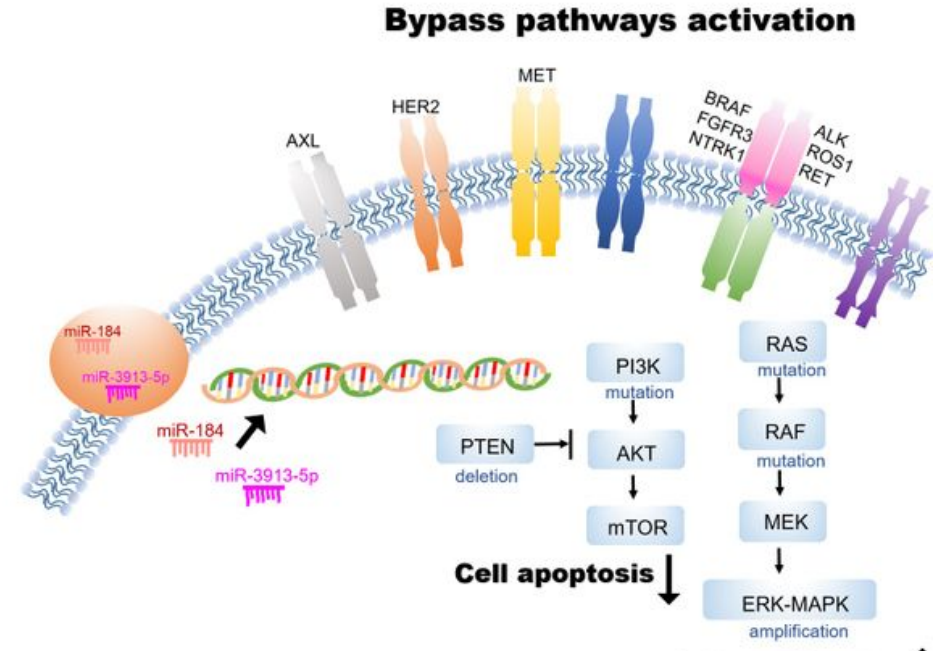

Cell proliferation $\uparrow$

Figure 5

Participation of exosomal miRNAs in osimertinib resistance through bypass activation mechanisms in NSCLC.
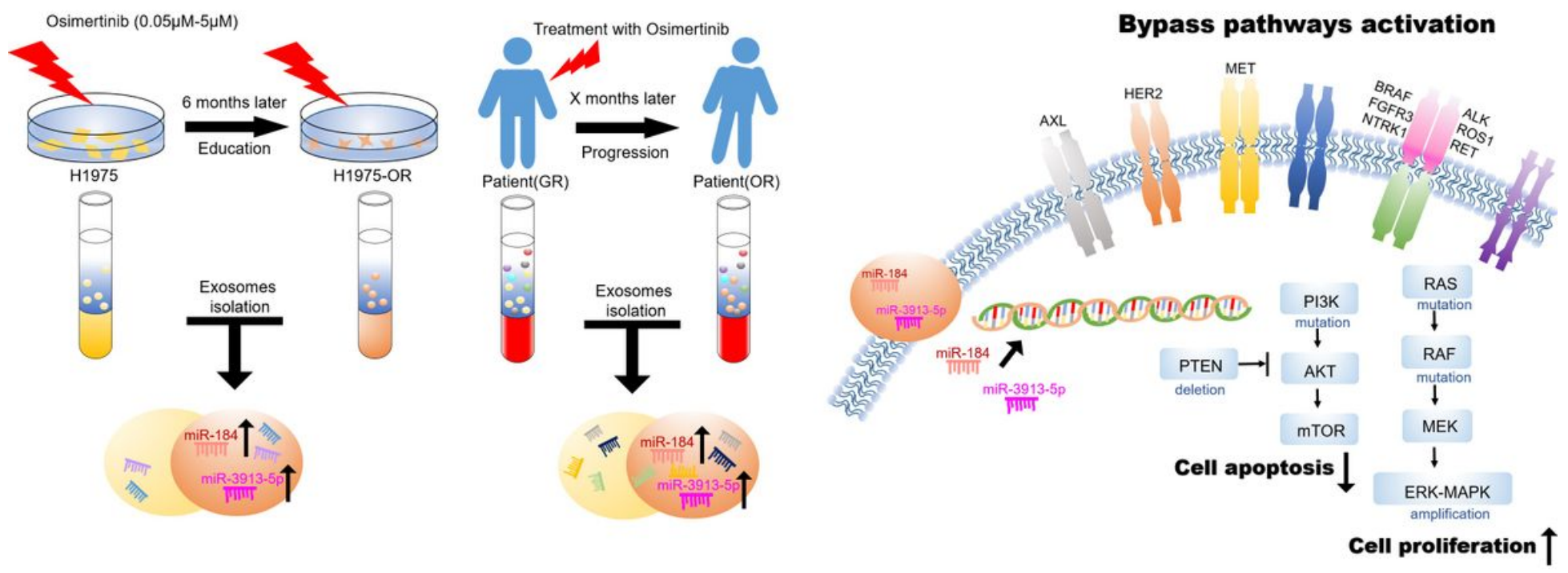

Figure 5

Participation of exosomal miRNAs in osimertinib resistance through bypass activation mechanisms in NSCLC. 

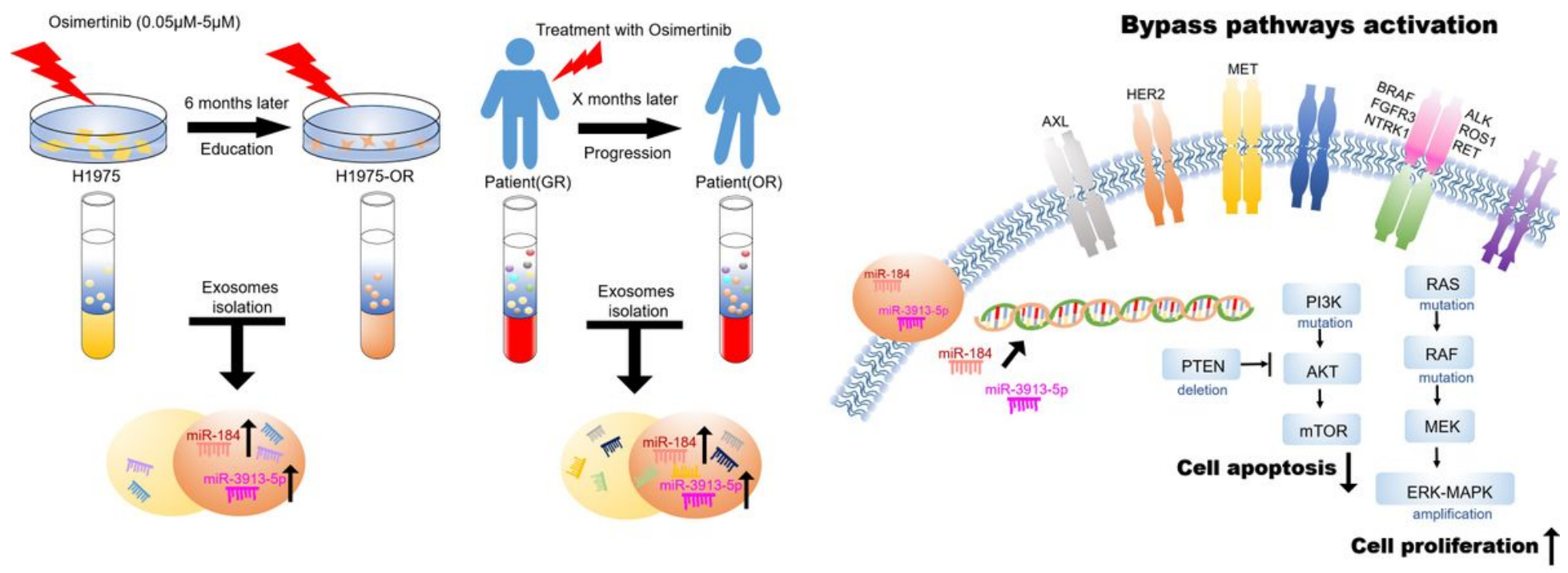

Figure 5

Participation of exosomal miRNAs in osimertinib resistance through bypass activation mechanisms in NSCLC.

\section{Supplementary Files}

This is a list of supplementary files associated with this preprint. Click to download.

- Supplementaldata.pdf

- Supplementaldata.pdf

- Supplementaldata.pdf 